\title{
In situ characterization of calcium fluxes in astrocytic mitochondria from the mouse striatum and hippocampus
}

\section{Taylor Huntington}

Texas A\&M University Health Science Center

Rahul Srinivasan ( $\square$ Rahul@tamu.edu )

Texas A\&M University Health Science Center

\section{Article}

Keywords: mitochondria, astrocytes, MCU, MAM, neurotransmitter, calcium, flux, neurodegeneration, striatum, hippocampus, CA1, dopamine, glutamate, D1 receptor, D2 receptor, mPTP

Posted Date: July 2nd, 2020

DOI: https://doi.org/10.21203/rs.3.rs-37291/v1

License: (c) (i) This work is licensed under a Creative Commons Attribution 4.0 International License.

Read Full License 


\section{Abstract}

Astrocytes govern critical aspects of brain function via $\mathrm{Ca} 2+$ signals, the majority of which associate with mitochondria. However, little is known with regard to in situ sources, kinetics or mechanisms of $\mathrm{Ca} 2+$ influx in astrocytic mitochondria. To address this knowledge gap, we expressed the genetically encoded calcium indicator, GCaMP6f within the mitochondrial matrix of adult mouse astrocytes in the dorsolateral striatum (DLS) and hippocampus (HPC). We found spontaneous Ca2+ events in astrocytic mitochondria with subcellular differences between somatic, branch, and branchlet mitochondria, as well as interregional differences between astrocytes in the DLS and HPC. We also found a strong dependency of spontaneous mitochondrial $\mathrm{Ca} 2+$ fluxes on endoplasmic reticulum stores, the surprising lack of a major role for the mitochondrial calcium uniporter, $\mathrm{MCU}$, and dual mitochondrial $\mathrm{Ca} 2+$ responses with multiple neurotransmitter agonists. Together, our findings provide a foundational understanding of mechanisms for $\mathrm{Ca} 2+$ influx in astrocytic mitochondria within disease-relevant brain regions.

\section{Introduction}

Once regarded as mere supporting cells, astrocytes have recently emerged as critical players in governing multiple aspects of brain physiology. Among their many functions, these cells modulate neural activity 1,2 , control synapse formation3, maintain $\mathrm{K}+$ homeostasis and neuronal excitability 4,5, regulate neurovasculature6,7, and provide $20 \%$ of the total energy required by the brain8,9.

The multi-faceted effects of astrocytes on nervous system function are thought to depend on spontaneous $\mathrm{Ca} 2+$ signals in astrocytic somata and the fine processes within their territories 10-14. Strong evidence for a central role played by astrocytic $\mathrm{Ca} 2+$ signals in regulating neural function comes from multiple reports showing that astrocytic $\mathrm{Ca} 2+$ signals are enhanced by behavioral stimuli such as forced locomotion 15 , or an air puff-induced startle response 16 , and are robustly modulated by drugs affecting the central nervous system, such as anesthetics17,18 and amphetamine19. Importantly, the disruption of $\mathrm{Ca} 2+$ fluxes specifically in astrocytes causes repetitive behaviors in mice20, potentiates short-term plasticity in the hippocampus21, and inhibits metabolic coupling between astrocytes and neurons22. Together these studies show that astrocytic $\mathrm{Ca} 2+$ signals are capable of influencing critical aspects of brain activity and consequently, behavioral outputs during health and disease. Therefore, developing an understanding of the mechanisms underlying astrocytic $\mathrm{Ca} 2+$ signals is important from a basic, as well as a translational perspective.

A recent study showed that the vast majority of spontaneous $\mathrm{Ca} 2+$ events within astrocytic processes in vivo occur due to brief periods of $\mathrm{Ca} 2+$ efflux through the mitochondrial permeability transition pore (mPTP), and are abnormally increased in a mouse model of amyotrophic lateral sclerosis 23 . This suggests that mitochondrial $\mathrm{Ca} 2+$ fluxes in astrocytes are not only critical for normal brain function, but can also significantly influence neurodegenerative processes. The central role played by astrocytic mitochondria during neurodegeneration is further highlighted by independent studies showing that disruption of astrocytic mitochondria exacerbates neurodegeneration in cerebellar Purkinje neurons24, 
attenuates neuroprotection following ischemia25, and even slows the recovery of mice from anesthesia26. Despite the many important roles ascribed to astrocytic mitochondria, and specifically to mitochondrial $\mathrm{Ca} 2+$ fluxes in astrocytes during health and disease, we know very little with regard to the subcellular characteristics of astrocytic mitochondria in situ, whether or not $\mathrm{Ca} 2+$ spontaneously fluxes into the mitochondria of astrocytes, and the extent to which astrocytic mitochondrial Ca2+ events respond to neurotransmitter agonists.

To address these broadly significant questions, we generated an adeno-associated virus (AAV)-based genetically encoded calcium indicator (GCaMP6f) specifically targeted to the matrix of astrocytic mitochondria and directly measured mitochondrial $\mathrm{Ca} 2+$ influx in striatal and hippocampal astrocytes in situ from adult mouse brain slices. Our experiments reveal that astrocytic mitochondria in the dorsolateral striatum (DLS) and hippocampus (HPC) display unique characteristics with regard to their morphological features, the kinetics of spontaneous mitochondrial $\mathrm{Ca} 2+$ events, sources and portals for entry of $\mathrm{Ca} 2+$ into mitochondria, and the response of astrocytic mitochondrial $\mathrm{Ca} 2+$ events to neurotransmitter agonists. These findings have important implications for developing a basic understanding of how mitochondrial $\mathrm{Ca} 2+$ events in astrocytes can influence normal brain physiology, and thereby contribute to a number of neuropathological processes, including neurodegeneration.

\section{Materials And Methods}

\section{Generation of AAV 2/5 GfaABC1D-mito-7-GCaMP6f}

The GfaABC1D-mito-7-GCaMP6f construct was generated by Vector Builder (Chicago, IL) using the Gateway cloning method27. Entry clones containing the mito-7 signaling sequence ( $87 \mathrm{bp})$, derived from COX8A and the GfaABC1D promoter ( $877 \mathrm{bp}$ ) were generated and recombined into a destination vector to create the construct. The GfaABC1D-mito-7-GCaMP6f cassette was then introduced into a pZac2.1 plasmid to create pZac2.1 GfaABC1D-mito-7-GCaMP6f, and this plasmid was used to generate the AAV 2/5 GfaABC1D-mito-7-GCaMP6f viral vector.

\section{Mice}

Male and female C57BL/6 WT breeders were obtained from Taconic and bred in house. Mice used for MCU-/- experiments were of a CD1 background and purchased from Texas A\&M Institute for Genomic Medicine (TIGM). MCU-/- mice were generated via gene trap method by integrating a retroviral trapping vector into the first intron of the CCDC109A locus28. WT CD1 littermates were used as controls for MCU-/experiments.

Mice were housed on a $12 \mathrm{~h}$ light/dark cycle with ad libitum access to food and water. For $\mathrm{Ca}^{2+}$ imaging, mice were injected with AAV2/5 GfaABC1D-mito-7-GCaMP6f at approximately 8 weeks old and imaged 3 weeks later. All animal experiments were conducted in accordance with Texas A\&M University IACUC guidelines. 


\section{Immunostaining}

For staining mouse DLS and HPC sections, mice were transcardially perfused with $10 \%$ formalin and brains were extracted. Brains were postfixed in $10 \%$ formalin overnight at $4^{\circ} \mathrm{C}$ and cryoprotected in $30 \%$ sucrose for $48-72 \mathrm{hrs} .40 \mu \mathrm{m}$ thick coronal sections were obtained using a cryostat microtome (Leica) and preserved in $0.01 \%$ sodium azide + PBS at $4^{\circ} \mathrm{C}$ until use. Immunohistochemistry was performed using previously published techniques 16,29 . Briefly, sections were washed $3 x$ for 10 min in $1 X$ TBS, then blocked for $1 \mathrm{hr}$ at RT in 1X TBS solution containing 5\% NGS + 0.25\% Triton-X-100. Sections were incubated overnight at $4^{\circ} \mathrm{C}$ in primary antibodies diluted in blocking solution. The following primary antibodies were used: chicken anti-GFP (1:2000; Abcam ab13970) and mouse anti-pyruvate dehydrogenase (PDH) (1:1000, Abcam ab110333). The following day sections were washed $3 x$ for 10 min each in 1X TBS and incubated with appropriate secondary antibodies in blocking solution for $2 \mathrm{hr}$ at RT. The following secondary antibodies were used: Alexa-488 goat anti-chicken (1:2000; Abcam ab150176) and Alexa-594 goat anti-mouse (1:2000; Abcam ab150120). Sections were rinsed 3x for 10 min in 1X TBS and then mounted on microscope slides in Fluoromount (Diagnostic Biosystems; K024) for imaging.

\section{Stereotaxic surgery}

Surgical procedures were conducted as previously described 16 . Briefly, surgeries were performed under general anesthesia using continuous isoflurane (induction at $5 \%$, maintenance at $1-2 \% \mathrm{vol} / \mathrm{vol}$ ) administered via a syringe injection system (Kent Scientific). Following head fixation on a stereotaxic frame (David Kopf Instruments, Tujunga CA), a 2-3 mm diameter craniotomy was performed using a highspeed dental drill (Foredom) and $0.9 \%$ saline was intermittently applied to reduce heating caused by the drill. Glass injection pipettes (World Precision Instruments, 1B100-4) were pulled using a Sutter P-2000 laser puller and beveled using a Narishige EG-45 grinder. $2 \times 10^{9}$ genome copies (gc) of AAV2/5 GfaABC1D-mito7- GCaMP6f was injected in a $2 \mu \mathrm{L}$ volume using a syringe pump (Harvard Apparatus). The injection pipette was left in place for $\sim 10 \mathrm{~min}$ after injection and then gradually withdrawn. Surgical wounds were closed with non-absorbable 5-0 sutures (Ethicon, 682G) and mice were sacrificed 20-23 days post-surgery for $\mathrm{Ca} 2+$ imaging. The injection coordinates for the DLS were as follows: anterior to bregma, $+0.9 \mathrm{~mm}$; lateral to bregma, $+1.8 \mathrm{~mm}$; and ventral to pial surface, $+2.5 \mathrm{~mm}$. Coordinates for the CA1 region of HPC were as follows: posterior to bregma, $-2.0 \mathrm{~mm}$; lateral to bregma, $+1.5 \mathrm{~mm}$; and ventral to pial surface, $-1.6 \mathrm{~mm}$.

\section{Ca2+ imaging in acute mouse brain slices}

$300 \mu \mathrm{m}$ thick coronal slices of either the DLS or HPC were cut in a slicing solution consisting of (in mM): 194 sucrose, $30 \mathrm{NaCl}, 4.5 \mathrm{KCl}, 1.2 \mathrm{NaH} 2 \mathrm{PO} 4,26 \mathrm{NaHCO}$, 10 D-glucose, and $1 \mathrm{MgCl} 2$ and saturated with $95 \% 02$ and $5 \% \mathrm{CO} 2$ (pH 7.2). Incubation and recording were performed in artificial cerebrospinal fluid (ACSF) comprising (in mM): $126 \mathrm{NaCl}, 2.5 \mathrm{KCl}, 1.24 \mathrm{NaH} 2 \mathrm{PO} 4,26 \mathrm{NaHCO}, 10 \mathrm{D}$-glucose, $2.4 \mathrm{CaCl}$, and $1.3 \mathrm{MgCl} 2$ saturated with 95\% 02 and $5 \% \mathrm{CO} 2\left(\mathrm{pH} \mathrm{7.4)}\right.$. Slices were incubated in ACSF at $34^{\circ} \mathrm{C}$ for $10 \mathrm{~min}$ 
and then incubated in ACSF with $150 \mathrm{nM}$ of MitoTracker Deep Red (MTDR) (Invitrogen) at $34^{\circ} \mathrm{C}$ for $1 \mathrm{hr}$, just prior to imaging. $150 \mathrm{nM}$ MTDR30,31 was used as an optimal concentration in acute slices and was determined as an appropriate concentration based on preliminary testing with concentrations ranging from $50 \mathrm{nM}-300 \mathrm{nM}$.

Imaging was performed as previously described 16 . Briefly, slices were imaged using an Olympus FV1200 upright laser-scanning confocal microscope with a 40X water immersion objective lens, numerical aperture (NA) of $0.8,488 \mathrm{~nm}$, and $633 \mathrm{~nm}$ laser lines. For each imaging session, the $488 \mathrm{~nm}$ line intensity was set at 10\% maximum output to visualize GCaMP6f fluorescence, and the $633 \mathrm{~nm}$ laser line at 1-5\% maximum output to visualize MTDR fluorescence. Confocal parameters (high voltage, gain, offset, laser power, and aperture diameter) were maintained constant for all GCaMP6f imaging and were optimized for MTDR during each imaging session. Ca2+ events were recorded at either $800 \mathrm{msec} /$ frame (Figs. 1, 310) or 1 frame/sec (FPS) (Fig. 2).

All drugs were bath perfused using a peristaltic pump (Harvard Apparatus) and the time taken for bath perfusion of drugs were set prior to each imaging session. Spontaneous activity in mitochondria was recorded for $5 \mathrm{~min}$ and drugs were sequentially applied with the order of application of drugs randomized for each slice. Concentrations of drugs applied were as follows: $300 \mu \mathrm{M}$ glutamate, $5 \mu \mathrm{M}$ SKF-38393, 10 $\mu \mathrm{M}$ quinpirole, $1 \mu \mathrm{M}$ or $10 \mu \mathrm{M}$ Ru265 (membrane permeable)32, and $20 \mu \mathrm{M}$ cyclopiazonic acid (CPA). 1 $\mu \mathrm{M}$ Ru265 was introduced to the bath, followed by $10 \mu \mathrm{M}$ Ru265 after a 10 min washout. For zero $\mathrm{Ca}^{2+}$ experiments $\mathrm{CaCl} 2$ was omitted from the recording buffer 16 . For CPA experiments, slices were incubated with $20 \mu \mathrm{M}$ CPA for 15 min prior to imaging and CPA was maintained in the bath during imaging. Washout times for each drug were as follows: glutamate (10 min), SKF-38393 (35 min), quinpirole (35 $\mathrm{min})$, zero Ca2+ (10 min), Ru265 (10 min), CPA (no washout possible).

\section{Data analyses}

Ca2+ signals were analyzed using MiniAnalysis program 6.0.7 (Synaptosoft) and Origin (2019, Origin Lab Corp.). Image analyses were performed using ImageJ version $1.52(\mathrm{NIH})$. Ca2+ imaging data were analyzed as previously described16. Briefly, image stacks were drift corrected in the $x-y$ direction using the Turboreg plugin in ImageJ. 1-5\% laser power at $633 \mathrm{~nm}$ caused significant photobleaching over the imaging period and this was corrected using EMBLtools (ImageJ plugin) with a frame-wise exponential fit.

Following drift and bleach correction MTDR labeled regions of interest (ROIs) were isolated using GEClquant as previously described 16 . We utilized an area constraint of 5-2000 $\mu \mathrm{m} 2$ for somatic and large territory mitochondria and 1-4 $\mu \mathrm{m} 2$ for small territory mitochondria. ROls obtained in this way were used to extract $\mathrm{Ca}^{2+}$ events from GfaABC1D-mito-7-GCaMP6f labelled astrocytic mitochondria. ROIs containing no $\mathrm{Ca} 2+$ events were manually screened and eliminated. $\mathrm{Ca} 2+$ event frequency (events/min), amplitude (dF/F), and half-width (s) were analyzed using Minianalysis 6.0.07 (Synaptosoft).

\section{Statistical analyses}


$\mathrm{Ca} 2+$ event measurements of frequency, amplitude and half widths were obtained from individually demarcated ROIs of mitochondria in the soma, branches or branchlets within individual astrocytes. Thus, each data point in the scatter plots represents one mitochondrial locus from one astrocyte. Averages for each of the Ca2+ event parameters were obtained from $3-4$ astrocytes per slice, $2-4$ slices per mouse, and between $4-17$ mice per condition. Exact sample sizes for each experiment are specified in the figure legends. Slices from male and female mice were pooled together since we did not observe sex differences in our experiments. Statistics were performed using Origin Lab. To test for statistical significance, data were first tested for normality using Shapiro-Wilk test, and in the case of datasets with normally distributed data, either student's unpaired t-test or paired t-test was used. Datasets containing non-normally distributed data were tested using Mann-Whitney (for unpaired data) or the Wilcoxon Signed Rank test (for paired data). Data were considered statistically significant if $p<0.05$. Statistical tests used for each experiment used are indicated in figure legends.

\section{Results}

\section{AAV 2/5 GfaABC1D-mito-7-GCaMP6f expresses GCaMP6f in astrocytic mitochondria}

To specifically express GCaMP6f in astrocytic mitochondria, we generated an adeno- associated viral vector (AAV) with the astrocyte-specific GfaABC1D promoter33 driving expression of GCaMP6f tagged to a mitochondrial mito-7 targeting sequence (AAV 2/5 GfaABC1D-mito-7-GCaMP6f). AAV 2/5 GfaABC1Dmito-7-GCaMP6f virus was stereotaxically injected into the DLS of WT C57BL/6 mice. Three weeks later, live striatal slices were obtained from AAV-injected mice, labeled with MTDR, and imaged using a confocal microscope (Fig 1a).

MTDR co-localized with AAV-expressed GCaMP6f in discrete punctate structures within the soma, proximal primary branches, and peripheral branchlets of astrocytes (Fig. 1b). To further confirm colocalization of GCaMP6f in the mitochondria of astrocytes, we immunostained a separate set of DLS sections from AAV 2/5 GfaABC1D-mito-7-GCaMP6f-injected mice with the mitochondrial matrix protein pyruvate dehydrogenase (PDH)34,35. These sections showed co-localization of PDH with GFP antibodylabeled GCaMP6f in DLS astrocytes (Supplementary Fig. 1). Thus, using two independent methods, i.e. live imaging of striatal slices with MTDR, and immunostaining with mitochondria-specific PDH, we confirmed that AAV 2/5 GfaABC1D-mito-7-GCaMP6f specifically expressed GCaMP6f in astrocytic mitochondria.

\section{The size of functional mitochondria in DLS astrocytes depends on their subcellular location}

We used the live DLS brain slices obtained from AAV 2/5 GfaABC1D-mito-7-GCaMP6f injected adult mice to demarcate punctate MTDR labeled ROIs that also co-express GCaMP6f in astrocytic mitochondria. Areas of GCaMP6f + MTDR labeled punctate structures were measured and segregated according to size. Spatially segregated mitochondria were observed in the somata, primary branches, and peripheral branchlets of all imaged astrocytes. Area analysis revealed significantly different sizes of mitochondria in the somata versus branches and branchlets of astrocytes (Fig. 1c). The largest mitochondria were 
somatic, with an average area of $21.6 \pm 2.3 \mu \mathrm{m} 2$. Mitochondria in astrocyte territories were significantly smaller than somatic mitochondria with average areas of $13.8 \pm 0.9 \mu \mathrm{m} 2$ for primary branch, and $1.7 \pm$ $0.7 \mu \mathrm{m} 2$ for peripheral branchlets (Fig. 1c). Thus, depending on their subcellular localization (somata versus territory), mitochondria in DLS astrocytes show clear variations in their size, with the largest mitochondria appearing in the somata, and the smallest ones in the most peripheral branchlets.

\section{Astrocytic mitochondria in the DLS show heterogenous spontaneous $\mathrm{Ca}+$ events}

Live DLS slices from adult mice expressing mito-7-GCaMP6f were imaged for spontaneous Ca2+ events in mitochondria. Spontaneous $\mathrm{Ca} 2+$ events were observed in all three types of mitochondria (somatic, primary branches, and peripheral branchlets) (Fig. 1d and Supplementary movie 1). Average Ca2+ event frequencies in all three mitochondria populations were similar (1.1 \pm 0.05 events/min), but interestingly, all mitochondria displayed a very discrete frequency distribution pattern, showing highly consistent increments of 0.25 events/min (Fig. 1e). In order to determine if discrete frequency patterns occurred because all mitochondria in a single astrocyte flux $\mathrm{Ca} 2+$ at a single specific frequency or if each astrocyte contained a mixture of mitochondrial $\mathrm{Ca} 2+$ event frequencies, we plotted the $\mathrm{Ca} 2+$ flux frequencies of individual mitochondria for each DLS astrocyte. We found that individual astrocytes display heterogenous mitochondrial $\mathrm{Ca} 2+$ event frequencies (Supplementary Fig. 2), suggesting a subcellular, rather than en masse regulation of astrocytic mitochondrial Ca2+ event frequencies in the DLS.

We found that amplitudes and half-widths of Ca2+ events in DLS astrocytes differed significantly among somatic, branch and branchlet mitochondria (Fig. 1e). Somatic mitochondria displayed the largest amplitude $(0.93 \pm 0.09 \mathrm{dF} / \mathrm{F})$, followed by secondary branchlet $(0.52 \pm 0.01 \mathrm{dF} / \mathrm{F})$ and primary branch mitochondria $(0.41 \pm 0.02 \mathrm{dF} / \mathrm{F})$. Ca2 + events in somatic mitochondria also demonstrated the longest half-width (3.65 $\pm 0.17 \mathrm{~s})$, followed by branch $(2.79 \pm 0.07 \mathrm{~s}$ ) and branchlet mitochondria (1.01 $\pm 0.04 \mathrm{~s})$. Thus, in addition to morphological heterogeneity, astrocytic mitochondria show specific differences in the kinetics of $\mathrm{Ca} 2+$ events, and this appears to be determined by the subcellular localization of mitochondria within an astrocyte.

\section{$\mathrm{Ca} 2+$ events in astrocytic mitochondria require endoplasmic reticulum (ER) Ca2+ stores}

We next assessed potential sources for spontaneous Ca2+ events in DLS astrocytic mitochondria. To empty ER Ca2+ stores, live DLS slices were exposed for $15 \mathrm{~min}$ to $20 \mu \mathrm{M}$ of the SERCA ATPase inhibitor, cyclopiazonic acid (CPA). We found that CPA caused a dramatic 4-fold decrease in Ca2+ event frequency for somatic, branch and branchlet mitochondria (Fig. 2a,b,d and Supplementary movie 2). Bath perfusion of slices with zero Ca2+ ACSF, however, did not alter mitochondrial Ca2+ events (Fig. 2c,e and Supplementary movie 3 ). The few remaining $\mathrm{Ca} 2+$ events after CPA showed significantly decreased amplitudes in all mitochondria (2.5-fold for soma and 1.5-fold for territory mitochondria), while halfwidths remained largely unchanged (Supplementary Fig. 3). By contrast, zero Ca2+ ACSF had minimal effect on mitochondrial $\mathrm{Ca} 2+$ event amplitudes and half-widths (Supplementary Fig. 3). Based on these data, we conclude that the ER is a major source of Ca2+ fluxes in DLS astrocytic mitochondria, with very little contribution from extracellular calcium. 
Having found that mitochondrial Ca2+ events in DLS astrocytes primarily depend on ER stores, we sought to determine whether the mitochondrial calcium uniporter, MCU36,37 is a major portal for entry of $\mathrm{Ca}^{2+}$ into astrocytic mitochondria. We injected AAV 2/5 GfaABC1D-mito-7- GCaMP6f into the DLS of MCU-/- mice in an outbred CD1 genetic background, which survives into adulthood despite the knockout of MCU28. Surprisingly, live DLS slices obtained from these mice displayed spontaneous astrocytic mitochondrial Ca2+ events that were indistinguishable from their WT littermates (Fig. 3a-c and Supplementary movie 4).

To address the possibility of compensatory mechanisms in CD1 MCU-/- mice38, a selective and membrane permeable MCU blocker, Ru26532, was bath perfused onto AAV 2/5- GfaABC1D-mito-7GCaMP6f injected DLS slices from WT C57BI6 mice. Exposure to either 1 or $10 \mu \mathrm{M}$ Ru265 did not inhibit mitochondrial Ca2+ event amplitudes or half-widths (Supplementary Fig. 4), but both concentrations of Ru265 caused a 25\% decrease in the inter- frequency interval for all mitochondrial subpopulations from 0.25 to 0.1 event/min (Fig. 3d-f). These data suggest that rather than being the primary portal for Ca2+ flux in astrocytic mitochondria, MCU likely regulates the frequency of mitochondrial Ca2+ events in astrocytes.

\section{Ca2+ events in mitochondria of DLS astrocytes are distinct from those in the HPC}

Astrocytes in the DLS possess a significantly different proteomic and transcriptional profile from the HPC39, and astrocyte populations have been shown to be as heterogenous as neurons40,41. Based on these findings, we asked if astrocytic mitochondria from these two brain regions also display heterogeneity. We compared the morphological profile and spontaneous $\mathrm{Ca} 2+$ event kinetics of astrocytic mitochondria from the DLS with those in the HPC.

The CA1 region in the HPC of WT C57BL/6 mice was stereotaxically injected with AAV2/5 GfaABC1Dmito-7-GCaMP6f. Live HPC slices from these mice were labeled with MTDR and imaged with a confocal microscope. Similar to DLS astrocytes, HPC astrocytes showed robust GCaMP6f expression that colocalized with MTDR to the soma, primary branches, and peripheral branchlets (Fig. 4a). Interestingly, somatic mitochondria in HPC astrocytes were 2-fold smaller than DLS astrocytes, while mitochondria in branches and branchlets were of similar size to DLS astrocytes (Fig. 4b).

We found that astrocytic mitochondria in the HPC showed spontaneous Ca2+ events (Fig. 4c and Supplementary movie 6). Irrespective of their localization to somata, branches or branchlets, all astrocytic mitochondria in the HPC displayed Ca2+ event frequencies that were half the frequency of Ca2+ events in DLS astrocytes (0.6 events/min for HPC versus 1.1 events/min for DLS) (Fig. 4d). Despite a lower average frequency of $\mathrm{Ca} 2+$ events in HPC astrocytic mitochondria, the interval between Ca2+ events in the HPC was always 0.25 event/min, which was similar to the DLS. By contrast, we found that amplitudes of $\mathrm{Ca} 2+$ events in HPC mitochondria were generally larger than those in the DLS for somatic, branch and branchlet mitochondria (Fig. 4e), but Ca2+ event half-widths were similar for both regions 
(Fig. 4f). Together, these data show that astrocytic mitochondria in the DLS differ from the HPC with regard to morphology, as well as the frequencies and amplitudes of $\mathrm{Ca} 2+$ fluxes.

\section{Mitochondrial Ca2+ events in DLS and HPC astrocytes show dual responses to glutamate}

Since the DLS and HPC receive glutamatergic input from the cortex42-45, we assessed the effects of glutamate on astrocytic mitochondrial $\mathrm{Ca} 2+$ events in both these brain regions.

Amplitudes and half-widths of astrocyte mitochondrial Ca2+ events in the DLS remained unchanged with bath application of $300 \mu \mathrm{M}$ glutamate (Supplementary Fig. 5a,b). However, glutamate exposure resulted in a dual effect on astrocytic mitochondrial Ca2+ event frequency. Bath application of $300 \mu \mathrm{M}$ glutamate decreased the Ca2+ event frequency by $43 \pm 10 \%$ in somatic, $46 \pm 7 \%$ in branch, and $54 \pm 5 \%$ in branchlet mitochondria, while other mitochondria within the same astrocytes showed an increase in event frequency by $57 \pm 10 \%$ in somatic, $54 \pm 7 \%$ in branch, and $46 \pm 5 \%$ in branchlet mitochondria (Figs. $5 a-c)$. Thus, each DLS astrocyte displayed a mixture of decreased or increased mitochondrial Ca2+ event frequency. For both effects of glutamate, viz. a decrease or increase in mitochondrial Ca2+ event frequency, glutamate invariably increased the dynamic range of mitochondrial $\mathrm{Ca} 2+$ event frequencies (Fig. 5c).

Similar to the DLS, bath application of glutamate to HPC slices did not alter mitochondrial Ca2+ event amplitudes and half-widths (Supplementary Fig. 5c,d), but caused a dual response in mitochondrial Ca2+ event frequency for all astrocytes that were imaged. For the HPC, we observed a decrease in frequency by $21 \pm 10 \%$ in somatic, $46 \pm 11 \%$ in branch, and $50 \pm 9 \%$ in branchlet mitochondria and an increase in frequency by $79 \pm 10 \%$ in somatic, $52 \pm 11 \%$ in branch, and $50 \pm 9 \%$ in branchlet mitochondria (Fig. $5 \mathrm{~d}-\mathrm{f}$ ). Similar to the DLS, glutamate eliminated the regular frequency spacing in all HPC astrocytic mitochondria.

\section{Mitochondrial Ca2+ events in DLS and HPC astrocytes show dual responses to dopaminergic D1 and D2 receptor agonists}

Dopaminergic neurons in the substantia nigra pars compacta (SNc) project to DLS and the HPC 46,47. Astrocytes in the DLS and HPC are therefore constantly exposed to dopamine in vivo, which would result in a sustained activation of D1 and D2 dopamine receptors in both brain regions. We assessed the effects of the D1-specific agonist, SKF-38393 and the D2 - specific agonist, quinpirole on mitochondrial $\mathrm{Ca} 2+$ events in astrocytes from the DLS and HPC. Similar to glutamate, bath application of $5 \mu \mathrm{M} \mathrm{SKF-}$ 38393 and $10 \mu \mathrm{M}$ quinpirole induced dual effects on Ca2+ event frequencies in somatic, branch, and branchlet mitochondria of DLS and HPC astrocytes (Figs. 6 and 7).

For the DLS, the D1-specific agonist, SKF-38393 decreased Ca2+ event frequencies by $29 \pm 9 \%$ in somatic, $49 \pm 8 \%$ in primary branches, and $38 \pm 6 \%$ in secondary branchlet mitochondria and increased $\mathrm{Ca} 2+$ event frequencies by $71 \pm 9 \%$ in somatic, $51 \pm 8 \%$ in primary branches, and $61 \pm 6 \%$ in secondary branchlet mitochondria of astrocytes (Fig. 6a-c). Astrocytic mitochondria in the HPC also displayed a 
dual response to SKF-38393 (Fig. 6d-f). SKF-38393 exposure decreased mitochondrial Ca2+ events in HPC astrocytes by $15 \pm 8 \%$ in somatic, $32 \pm 7 \%$ in primary branches, and $30 \pm 4 \%$ in secondary branchlets, and increased mitochondrial $\mathrm{Ca} 2+$ events by $83 \pm 8 \%$ in somatic, $68 \pm 7 \%$ in primary branches, and $70 \pm 4 \%$ in secondary branchlets.

The D2-specific agonist, quinpirole decreased Ca2+ event frequencies in DLS astrocytic mitochondria by $45 \pm 11 \%$ in somatic, $51 \pm 8 \%$ in primary branches, and $50 \pm 5 \%$ in secondary branchlet mitochondria, but increased Ca2+ event frequencies in other mitochondria by $55 \pm 11 \%$ in somatic, $49 \pm 8 \%$ in primary branches, and $52 \pm 5 \%$ in secondary branchlets (Figs. 7a-c). In the HPC, quinpirole caused a decrease in mitochondrial $\mathrm{Ca} 2+$ events by $11 \pm 8 \%$ in somatic, $37 \pm 7 \%$ in primary branches, and $30 \pm 5 \%$ in secondary branchlets and an increase in $\mathrm{Ca} 2+$ events of other mitochondria from the same HPC astrocytes by $89 \pm 8 \%$ in somatic, $63 \pm 7 \%$ in primary branches, and $70 \pm 5 \%$ in secondary branchlets (Fig. 7d-f). Neither SKF-38393 nor quinpirole altered amplitudes and half-widths of mitochondrial Ca2+ events in either the DLS or the HPC (Supplementary Figs. 6,7). However, regardless of whether they caused a decrease or an increase in mitochondrial Ca2+ event frequencies, both SKF-38393 and quinpirole dramatically increased the dynamic range of $\mathrm{Ca} 2+$ event frequencies in all astrocytic mitochondria from the DLS and HPC (Figs. $6 \mathrm{c}$ and 7c).

In summary, these data show that activating either glutamate or D1 and D2 neurotransmitter receptors in DLS and HPC astrocytes causes a dual response in mitochondrial Ca2+ event frequency. Thus, while some mitochondria show a decrease in frequency, other mitochondria in the same astrocyte robustly increase Ca2+ event frequencies.

\section{Mitochondrial Ca2+ responses to neurotransmitter receptor agonists do not depend on MCU}

We assessed the effect of glutamate, SKF-38393, and quinpirole on the mitochondrial Ca2+ event frequency in DLS astrocytes from MCU-/- mice. Following exposure to all three agonists (glutamate, SKF38393, and quinpirole), astrocytes from MCU-/- mice displayed dual responses of mitochondrial Ca2+ event frequencies and dramatic changes in frequency distributions of mitochondrial $\mathrm{Ca} 2+$ events that were indistinguishable from WT littermate controls (Figs. 8-10). These data suggest that MCU does not play a role in neurotransmitter-induced responses of astrocytic mitochondrial Ca2+ events.

\section{Discussion}

We generated an AAV construct, AAV2/5 GfaABC1D-mito-7-GCaMP6f, to measure the influx of Ca2+ into astrocytic mitochondria from DLS and HPC brain slices. Our experiments reveal several unique features in astrocytic mitochondria, such as: (i) Significant differences in mitochondrial size based on subcellular location, as well as the brain region in which the astrocyte resides, (ii) Divergence of spontaneous $\mathrm{Ca} 2+$ event kinetics in somatic, branch, and branchlet mitochondria, (iii) Strong dependence of mitochondrial $\mathrm{Ca} 2+$ fluxes on ER Ca2+ stores, (iv) The surprising lack of a major role for the mitochondrial calcium uniporter, $\mathrm{MCU}$ in $\mathrm{Ca} 2+$ fluxes, and (v) Dual responses of $\mathrm{Ca} 2+$ event frequencies to multiple 
neurotransmitter agonists. Taken together, these findings provide a comprehensive foundational understanding of mitochondrial $\mathrm{Ca} 2+$ fluxes in astrocytes within disease-relevant regions of the brain.

Using AAV-expressed GfaABC1D-mito-7-GCaMP6f co-labeled with MTDR, we found that DLS and HPC astrocytes possess distinct sizes of mitochondria in their soma, branches, and branchlets. The largest mitochondria localized to astrocytic soma, while the smallest ones were observed in peripheral secondary branchlets, and intermediate size mitochondria localized to primary branches of astrocyte territories (Figs. 1c and 4b). This finding is in agreement with previous studies showing similar distributions of mitochondria in cortical astrocytes23,48. Notably, somatic mitochondria in HPC astrocytes were half the size of those in the soma of DLS astrocytes (Fig. 4b), which likely represents a functional rather than a morphological adaptation. Indeed, despite having similar somatic sizes, the number of neuronal cell bodies contacted by astrocytes in the DLS is greater than the CA1 region in the HPC39. Thus, somatic mitochondria in astrocytes within these two brain regions might cater to the specific energy requirements of neuronal somata rather than individual synapses, while mitochondria in branches and branchlets likely respond to activity within individual synapses.

All of the GCaMP6f-expressing mitochondria in DLS and HPC astrocytes displayed robust spontaneous $\mathrm{Ca} 2+$ events. When compared to $\mathrm{Ca} 2+$ events in somata, we found that branch and branchlet mitochondria showed significantly smaller amplitudes and shorter half-widths (Figs. 1e and 4d-f). In addition, we observed a highly structured frequency distribution of spontaneous mitochondrial $\mathrm{Ca} 2+$ events in DLS and HPC astrocytes (Fig. 4d). For each of the mitochondrial loci analyzed, we found that $\mathrm{Ca} 2+$ events occurred at a single frequency not determined by subcellular location, and when analyzed in the context of individual astrocytes, mitochondrial $\mathrm{Ca} 2+$ events displayed a mixture of frequencies rather than one uniform frequency. Because mitochondrial $\mathrm{Ca} 2+$ is a primary determinant of oxidative phosphorylation and the ability to generate ATP49-51, we suggest that there is a quantitative correlation between subcellular ATP generation in astrocytes and the magnitude of $\mathrm{Ca} 2+$ events in the soma versus territories. Thus, unlike the heterogenous mitochondrial $\mathrm{Ca} 2+$ events in astrocyte territories, which would cater to small scale synaptic activity, the larger, more invariant $\mathrm{Ca} 2+$ events in somatic mitochondria generate more ATP for regulating the baseline activity of multiple neurons in close proximity to any given astrocyte. Apart from these subcellular differences, we also observed region-specific differences in the kinetics of spontaneous astrocytic mitochondrial Ca2+ events. Mitochondria in all sub-compartments of HPC astrocytes fluxed Ca2+ at a 2-fold lower frequency than astrocytes in the DLS (Fig. 4d). Our simplest interpretation for this finding is that when compared to the DLS, neurons in the hippocampus possess lower levels of baseline activity, which would correlate with the amount of astrocyte-generated ATP, and the magnitude of mitochondrial $\mathrm{Ca} 2+$ events in astrocytes from each of these brain structures.

We did not directly assess the directionality of spontaneous astrocytic mitochondrial Ca2+ fluxes. However, because the mito-7 signaling sequence would cause GCaMP6f to localize to the inner mitochondrial matrix, one can assume that GCaMP6f detects an influx of $\mathrm{Ca} 2+$ into the inner mitochondrial matrix, rather than the efflux of $\mathrm{Ca} 2+$ from mitochondria. To determine subcellular sources for Ca2+ influx into mitochondria in DLS astrocytes, we sequentially depleted extracellular and ER Ca2+ 
stores. Depleting extracellular Ca2+ did not alter mitochondrial Ca2+ events (Fig. 2c,e). By contrast, emptying ER Ca2+ stores with the SERCA ATPase pump inhibitor, CPA almost completely abolished Ca2+ events (Fig. 2b,d), suggesting that Ca2+ stores in the ER serve as a major source for spontaneous $\mathrm{Ca} 2+$ influx into astrocytic mitochondria. Calcium transfer from the ER to mitochondria likely occurs via physical domains, known as mitochondria-associated membranes (MAMs), which physically and functionally link the ER with mitochondria52,53. Strong evidence for the presence of MAMs in astrocytes comes from a recent study showing that astrocytic mitochondria extensively contact the ER through mitofusin tethering domains54. Although MAMs are enriched in ER-localized inositol triphosphate type 2 (IP3R2) receptors55, a previous study has shown that astrocytic mitochondrial $\mathrm{Ca}$ 2+ fluxes are independent of IP3R223. An alternative possibility is that $\mathrm{Ca} 2+$ transfer from the ER to mitochondria occurs through other isoforms of IP3 receptors, and ER-localized ryanodine receptors (RyRs)56,57. Thus, we surmise that spontaneous $\mathrm{Ca} 2+$ events in astrocytic mitochondria occur at MAMs, and most likely involve Ca2+ transfer to mitochondria via ER-localized isoforms of IP3 and RyR receptors.

Having confirmed the ER as a primary source of $\mathrm{Ca} 2+$ for astrocytic mitochondria, we sought to determine the extent to which the mitochondrial calcium uniporter, MCU plays a role for $\mathrm{Ca} 2+$ entry into the inner mitochondrial matrix. To this end, we examined mitochondrial $\mathrm{Ca} 2+$ events in astrocytes from MCU-/- mice, and much to our surprise, found that Ca2+ events in DLS astrocytes from MCU-/- mice were indistinguishable from WT littermates (Fig. 3a-c). Compensatory mechanisms in MCU-/- mice are unlikely to play a role because exposure to the MCU-specific inhibitor, Ru265, failed to inhibit mitochondrial Ca2+ fluxes in DLS astrocytes from WT mice (Fig. 3d-f). We observed that the Ru265-mediated inhibition of MCU significantly altered the frequency distribution of $\mathrm{Ca} 2+$ events, which suggests that rather than acting as a major portal for Ca2+ entry into mitochondria, as observed in other cells36, MCU in astrocytes may regulate specific aspects of mitochondrial $\mathrm{Ca} 2+$ flux kinetics. Our data further indicate that the regulatory role of MCU may be restricted to spontaneous $\mathrm{Ca} 2+$ events in mitochondria, because when compared to WT littermates, exposure to neurotransmitter agonists did not alter mitochondrial $\mathrm{Ca} 2+$ fluxes in astrocytes from MCU-/- mice. In the absence of $\mathrm{MCU}$ as a major portal for $\mathrm{Ca} 2+$ influx into the matrix of astrocytic mitochondria, one candidate molecule to consider is the mitochondrial sodium calcium exchanger, NCLX. Evidence for an important role of NCLX comes from a study showing that NCLX mediates spontaneous Ca2+ oscillations in mitochondria by sequentially alternating between forward, $\mathrm{Ca} 2+$ efflux and reverse $\mathrm{Ca} 2+$ influx transport modes58. In addition, siRNA-mediated or pharmacological inhibition of NCLX alters cytosolic Ca2+ fluxes and ATP responses in astrocytes59. These previous studies, along with our results strongly indicate that rather than $\mathrm{MCU}$, the mitochondrial transporter, NCLX may be the primary portal for $\mathrm{Ca} 2+$ influx into the inner matrix of astrocytic mitochondria.

Finally, we assessed the response of mitochondrial Ca2+ events in astrocytes to neurotransmitters by bath perfusing DLS and HPC brain slices with glutamate, the D1 receptor-specific agonist SKF-38393, or the D2 receptor-specific agonist quinpirole. Each of these neurotransmitter receptor agonists caused dual responses in mitochondrial $\mathrm{Ca} 2+$ flux frequencies within the somata, branches, and branchlets of DLS and HPC astrocytes (Figs. 5-10). Specifically, while a proportion of astrocytic mitochondria increased their 
frequency, $\mathrm{Ca} 2+$ event frequencies in other mitochondria from the same astrocyte were significantly reduced. Although mitochondrial $\mathrm{Ca} 2+$ event frequencies were altered by all tested neurotransmitter receptor agonists, their amplitudes and half widths remained largely unchanged (Supplementary Figs. 57), which suggests that $\mathrm{Ca} 2+$ flux frequencies in mitochondria are a primary determinant of astrocyte responses to neurotransmitters. In line with our finding, a previous study utilized bulk loaded Fluo-3 AM into HPC astrocytes in situ, and showed that dopamine causes a similar dual response in astrocytic Ca2+ signals60. Here, we show that these previously reported dopamine-induced dual responses are in fact mediated by changes in the frequency of $\mathrm{Ca} 2+$ events in the mitochondria of astrocytes. Conceivably, $\mathrm{Ca} 2+$ event frequencies for any one mitochondrion in an astrocyte territory are likely to be synchronized with one or more associated microcircuits. This can cause neurotransmitters to independently alter $\mathrm{Ca} 2+$ event frequencies, based on energy requirements for a particular microcircuit linked to one or more astrocytic mitochondria. Divergent neurotransmitter-induced changes in the frequency of mitochondrial $\mathrm{Ca} 2+$ events within the same astrocyte would not only modulate ATP generation in microcircuits, but can also cause genetic and epigenetic changes in the astrocytes themselves61. Thus, responses of astrocytic mitochondrial $\mathrm{Ca} 2+$ fluxes to sustained neurotransmitter release will, in the long-term, alter signaling mechanisms between astrocytes and neurons.

In conclusion, our study shows that astrocytic mitochondria possess a unique physiological profile in the form of spontaneous $\mathrm{Ca} 2+$ fluxes, with significant differences from other cells in their kinetics and portals of $\mathrm{Ca} 2+$ entry. We also report subcellular and inter-regional differences, as well as dual responses of astrocytic mitochondrial $\mathrm{Ca} 2+$ fluxes to neurotransmitter agonists. Together, these results provide a strong mechanistic foundation for understanding how $\mathrm{Ca} 2+$ fluxes in the mitochondria of astrocytes change during neurodegeneration, stroke, or aging. We predict that our findings will enable specific manipulations of $\mathrm{Ca} 2+$ fluxes in astrocytic mitochondria as an effective treatment strategy for multiple neurological conditions.

\section{Declarations}

Acknowledgements: Supported by a pre-doctoral award from the National Science Foundation Graduate Research Fellowships Program (NSF-GRFP) to TEH and a grant from the Texas A\&M University (TAMU) Clinical Science and Translational Research (CSTR) pilot program to RS. We thank the Texas A\&M Institute for Genomic Medicine (TIGM) for providing MCU-/- mice and Dr. Phillip A. West (TAMU College of Medicine) for useful discussions on mitochondrial biology. We thank Dr. Madesh Muniswamy (University of Texas Health Science Center at San Antonio) and Dr. Justin J. Wilson (Cornell University) for providing the MCU- specific inhibitor, Ru265.

Conflict of interest: Authors declare no competing financial interests

Author contributions: TEH performed all experiments, analyzed data, and contributed to writing and editing the manuscript. RS trained and supervised TEH, coordinated and designed experiments, provided resources and funding, wrote and edited the manuscript. 


\section{References}

1. Halassa, M. M. \& Haydon, P. G. Integrated brain circuits: astrocytic networks modulate neuronal activity and behavior. Annu Rev Physio/ 72, 335-355, doi:10.1146/annurev- physiol-021909-135843 (2010).

2. Papouin, T., Dunphy, J., Tolman, M., Foley, J. C. \& Haydon, G. Astrocytic control of synaptic function. Philos Trans R Soc Lond B Biol Sci 372, doi:10.1098/rstb.2016.0154 (2017).

3. Chung, W. S., Allen, N. J. \& Eroglu, C. Astrocytes Control Synapse Formation, Function, and Elimination. Cold Spring Harb Perspect Biol 7, a020370, doi:10.1101/cshperspect.a020370 (2015).

4. Olsen, M. et al. New Insights on Astrocyte Ion Channels: Critical for Homeostasis and Neuron-Glia Signaling. J Neurosci 35, 13827-13835, doi:10.1523/JNEUROSCI.2603- 15.2015 (2015).

5. Bellot-Saez, , Kekesi, O., Morley, J. W. \& Buskila, Y. Astrocytic modulation of neuronal excitability through K(+) spatial buffering. Neurosci Biobehav Rev 77, 87-97, doi:10.1016/j.neubiorev.2017.03.002 (2017).

6. MacVicar, B. A. \& Newman, E. A. Astrocyte regulation of blood flow in the brain. Cold Spring Harb Perspect Bio/ 7, doi:10.1101/cshperspect.a020388 (2015).

7. Mishra, A. Binaural blood flow control by astrocytes: listening to synapses and the vasculature. $J$ Physio/ 595, 1885-1902, doi:10.1113/JP270979 (2017).

8. Bluml, S., Moreno-Torres, A., Shic, F., Nguy, C. H. \& Ross, B. D. Tricarboxylic acid cycle of glia in the in vivo human brain. NMR Biomed 15, 1-5, doi:10.1002/nbm.725 (2002).

9. Gollihue, J. \& Norris, C. M. Astrocyte mitochondria: Central players and potential therapeutic targets for neurodegenerative diseases and injury. Ageing Res Rev 59, 101039, doi:10.1016/j.arr.2020.101039 (2020).

10. Volterra, A., Liaudet, N. \& Savtchouk, Astrocyte $\mathrm{Ca}(2)(+)$ signalling: an unexpected complexity. Nat Rev Neurosci 15, 327-335, doi:10.1038/nrn3725 (2014).

11. Khakh, B. S. \& Sofroniew, M. V. Diversity of astrocyte functions and phenotypes in neural circuits. Nat Neurosci 18, 942-952, doi:10.1038/nn.4043 (2015).

12. Bazargani, N. \& Attwell, D. Astrocyte calcium signaling: the third wave. Nat Neurosci 19, 182-189, doi:10.1038/nn.4201 (2016).

13. Wang, , He, Y., Sejnowski, T. J. \& Yu, X. Brain-state dependent astrocytic $\mathrm{Ca}(2+)$ signals are coupled to both positive and negative BOLD-fMRI signals. Proc Natl Acad Sci U S A 115, E1647-E1656, doi:10.1073/pnas.1711692115 (2018).

14. Guerra-Gomes, S., Sousa, N., Pinto, L. \& Oliveira, J. F. Functional Roles of Astrocyte Calcium Elevations: From Synapses to Behavior. Front Cell Neurosci 11, 427, doi:10.3389/fncel.2017.00427 (2017).

15. Paukert, M. et al. Norepinephrine controls astroglial responsiveness to local circuit Neuron 82, $1263-$ 1270, doi:10.1016/j.neuron.2014.04.038 (2014). 
16. Srinivasan, R. et al. $\mathrm{Ca}(2+)$ signaling in astrocytes from Ip3r2(-/-) mice in brain slices and during startle responses in vivo. Nat Neurosci 18, 708-717, doi:10.1038/nn.4001 (2015).

17. Nimmerjahn, A., Mukamel, E. A. \& Schnitzer, M. J. Motor behavior activates Bergmann glial networks. Neuron 62, 400-412, doi:10.1016/j.neuron.2009.03.019 (2009).

18. Thrane, S. et al. General anesthesia selectively disrupts astrocyte calcium signaling in the awake mouse cortex. Proc Natl Acad Sci U S A 109, 18974-18979, doi:10.1073/pnas.1209448109 (2012).

19. Corkrum, M. et al. Dopamine-Evoked Synaptic Regulation in the Nucleus Accumbens Requires Astrocyte Activity. Neuron 105, 1036-1047 e1035, doi:10.1016/j.neuron.2019.12.026 (2020).

20. Yu, X. et al. Reducing Astrocyte Calcium Signaling In Vivo Alters Striatal Microcircuits and Causes Repetitive Behavior. Neuron 99, 1170-1187 e1179, doi:10.1016/j.neuron.2018.08.015 (2018).

21. Sibille, J., Zapata, J., Teillon, J. \& Rouach, N. Astroglial calcium signaling displays short- term plasticity and adjusts synaptic efficacy. Front Cell Neurosci 9, 189, doi:10.3389/fncel.2015.00189 (2015).

22. Murphy-Royal, C. et al. Stress gates an astrocytic energy reservoir to impair synaptic plasticity. Nat Commun 11, 2014, doi:10.1038/s41467-020-15778-9 (2020).

23. Agarwal, A. et al. Transient Opening of the Mitochondrial Permeability Transition Pore Induces Microdomain Calcium Transients in Astrocyte Processes. Neuron 93, 587-605 e587, doi:10.1016/j.neuron.2016.12.034 (2017).

24. Murru, S. et al. Astrocyte-specific deletion of the mitochondrial m-AAA protease reveals glial contribution to neurodegeneration. Glia 67, 1526-1541, doi:10.1002/glia.23626 (2019).

25. Shih, K. \& Robinson, M. B. Role of Astrocytic Mitochondria in Limiting Ischemic Brain Injury? Physiology (Bethesda) 33, 99-112, doi:10.1152/physiol.00038.2017 (2018).

26. Ramadasan-Nair, , Hui, J., Itsara, L. S., Morgan, P. G. \& Sedensky, M. M. Mitochondrial Function in Astrocytes Is Essential for Normal Emergence from Anesthesia in Mice. Anesthesiology 130, 423-434, doi:10.1097/ALN.0000000000002528 (2019).

27. Katzen, F. Gateway ((R)) recombinational cloning: a biological operating system. Expert Opin Drug Discov 2, 571-589, doi:10.1517/17460441.2.4.571 (2007).

28. Pan, X. et al. The physiological role of mitochondrial calcium revealed by mice lacking the mitochondrial calcium uniporter. Nat Cell Bio/ 15, 1464-1472, doi:10.1038/ncb2868 (2013).

29. Srinivasan, R. et al. New Transgenic Mouse Lines for Selectively Targeting Astrocytes and Studying Calcium Signals in Astrocyte Processes In Situ and In Vivo. Neuron 92, 1181-1195, doi:10.1016/j.neuron.2016.11.030 (2016).

30. Leonard, A. P. et al. Quantitative analysis of mitochondrial morphology and membrane potential in living cells using high-content imaging, machine learning, and morphological binning. Biochim Biophys Acta 1853, 348-360, doi:10.1016/j.bbamcr.2014.11.002 (2015).

31. Shu, et al. The effect of fluoxetine on astrocyte autophagy flux and injured mitochondria clearance in a mouse model of depression. Cell Death Dis 10, 577, doi:10.1038/s41419- 019-1813-9 (2019). 
32. Woods, J. et al. A Selective and Cell-Permeable Mitochondrial Calcium Uniporter (MCU) Inhibitor Preserves Mitochondrial Bioenergetics after Hypoxia/Reoxygenation Injury. ACS Cent Sci 5, 153-166, doi:10.1021/acscentsci.8b00773 (2019).

33. Lee, Y., Messing, A., Su, M. \& Brenner, M. GFAP promoter elements required for region- specific and astrocyte-specific expression. Glia 56, 481-493, doi:10.1002/glia.20622 (2008).

34. Berthet, A. et al. Loss of mitochondrial fission depletes axonal mitochondria in midbrain dopamine neurons. J Neurosci 34, 14304-14317, doi:10.1523/JNEUROSCI.0930-14.2014 (2014).

35. Yamada, T., Adachi, Y., Yanagawa, T., lijima, M. \& Sesaki, H. p62/sequestosome-1 knockout delays neurodegeneration induced by Drp1 loss. Neurochem Int 117, 77-81, doi:10.1016/j.neuint.2017.05.012 (2018).

36. De Stefani, D., Raffaello, A., Teardo, E., Szabo, I. \& Rizzuto, R. A forty-kilodalton protein of the inner membrane is the mitochondrial calcium uniporter. Nature 476, 336-340, doi:10.1038/nature10230 (2011).

37. Baughman, J. M. et al. Integrative genomics identifies MCU as an essential component of the mitochondrial calcium uniporter. Nature 476, 341-345, doi:10.1038/nature10234 (2011).

38. Murphy, E. et al. Unresolved questions from the analysis of mice lacking MCU Biochem Biophys Res Commun 449, 384-385, doi:10.1016/j.bbrc.2014.04.144 (2014).

39. Chai, H. et al. Neural Circuit-Specialized Astrocytes: Transcriptomic, Proteomic, Morphological, and Functional Evidence. Neuron 95, 531-549 e539, doi:10.1016/j.neuron.2017.06.029 (2017).

40. John Lin, C. C. et al. Identification of diverse astrocyte populations and their malignant analogs. Nat Neurosci 20, 396-405, doi:10.1038/nn.4493 (2017).

41. Batiuk, M. Y. et al. Identification of region-specific astrocyte subtypes at single cell resolution. Nat Commun 11, 1220, doi:10.1038/s41467-019-14198-8 (2020).

42. Graves, A. R. et al. Hippocampal pyramidal neurons comprise two distinct cell types that are countermodulated by metabotropic receptors. Neuron 76, 776-789, doi:10.1016/j.neuron.2012.09.036 (2012).

43. Arszovszki, , Borhegyi, Z. \& Klausberger, T. Three axonal projection routes of individual pyramidal cells in the ventral CA1 hippocampus. Front Neuroanat 8, 53, doi:10.3389/fnana.2014.00053 (2014).

44. Kim, H. J., Lee, J. H., Yun, K. \& Kim, J. H. Alterations in Striatal Circuits Underlying Addiction-Like Behaviors. Mol Cells 40, 379-385, doi:10.14348/molcells.2017.0088 (2017).

45. Fino, , Vandecasteele, M., Perez, S., Saudou, F. \& Venance, L. Region-specific and state- dependent action of striatal GABAergic interneurons. Nat Commun 9, 3339, doi:10.1038/s41467-018-05847-5 (2018).

46. Thompson, H., Grealish, S., Kirik, D. \& Bjorklund, A. Reconstruction of the nigrostriatal dopamine pathway in the adult mouse brain. Eur J Neurosci 30, 625-638, doi:10.1111/j.14609568.2009.06878.x (2009). 
47. Jeon, S. G., Kim, Y. J., Kim, K. A., Mook-Jung, \& Moon, M. Visualization of Altered Hippocampal Connectivity in an Animal Model of Alzheimer's Disease. Mol Neurobio/ 55, 7886-7899, doi:10.1007/s12035-018-0918-y (2018).

48. Jackson, J. G. \& Robinson, M. B. Regulation of mitochondrial dynamics in astrocytes: Mechanisms, consequences, and unknowns. Glia 66, 1213-1234, doi:10.1002/glia.23252 (2018).

49. Griffiths, E. J. \& Rutter, G. A. Mitochondrial calcium as a key regulator of mitochondrial ATP production in mammalian cells. Biochim Biophys Acta 1787, 1324-1333, doi:10.1016/j.bbabio.2009.01.019 (2009).

50. Tarasov, A. I., Griffiths, E. J. \& Rutter, G. A. Regulation of ATP production by mitochondrial Ca(2+). Cell Calcium 52, 28-35, doi:10.1016/j.ceca.2012.03.003 (2012).

51. Boyman, , Karbowski, M. \& Lederer, W. J. Regulation of Mitochondrial ATP Production: $\mathrm{Ca}(2+)$ Signaling and Quality Control. Trends Mol Med 26, 21-39, doi:10.1016/j.molmed.2019.10.007 (2020).

52. Csordas, G. et al. Structural and functional features and significance of the physical linkage between ER and mitochondria. J Cell Bio/ 174, 915-921, doi:10.1083/jcb.200604016 (2006).

53. Vance, J. E. MAM (mitochondria-associated membranes) in mammalian cells: lipids and beyond. Biochim Biophys Acta 1841, 595-609, doi:10.1016/j.bbalip.2013.11.014 (2014).

54. Gbel, J. et al. Mitochondria-Endoplasmic Reticulum Contacts in Reactive Astrocytes Promote Vascular Remodeling. Cell Metab 31, 791-808 e798, doi:10.1016/j.cmet.2020.03.005 (2020).

55. Bartok, A. et al. IP3 receptor isoforms differently regulate ER-mitochondrial contacts and local calcium transfer. Nat Commun 10, 3726, doi:10.1038/s41467-019-11646-3 (2019).

56. Okubo, Y. et al. Inositol 1,4,5-trisphosphate receptor type 2-independent $\mathrm{Ca}(2+)$ release from the endoplasmic reticulum in astrocytes. Glia 67, 113-124, doi:10.1002/glia.23531 (2019).

57. Okubo, \& lino, M. Visualization of astrocytic intracellular Ca(2+) mobilization. J Physio/ 598, 16711681, doi:10.1113/JP277609 (2020).

58. Samanta, , Mirams, G. R. \& Parekh, A. B. Sequential forward and reverse transport of the $\mathrm{Na}(+)$ $\mathrm{Ca}(2+)$ exchanger generates $\mathrm{Ca}(2+)$ oscillations within mitochondria. Nat Commun $\mathbf{9}, 156$, doi:10.1038/s41467-017-02638-2 (2018).

59. Parnis, J. et al. Mitochondrial exchanger NCLX plays a major role in the intracellular Ca2+ signaling, gliotransmission, and proliferation of astrocytes. J Neurosci 33, 7206-7219, doi:10.1523/JNEUROSCI.5721-12.2013 (2013).

60. Jennings, A. et al. Dopamine elevates and lowers astroglial $\mathrm{Ca}(2+)$ through distinct pathways depending on local synaptic circuitry. Glia 65, 447-459, doi:10.1002/glia.23103 (2017).

61. Lombardi, A. A. et al. Mitochondrial calcium exchange links metabolism with the epigenome to control cellular differentiation. Nat Commun 10, 4509, doi:10.1038/s41467-019-12103-x (2019).

\section{Figures}


a -GfaABC1D mito7 3xGGGS-GCaMP6f-

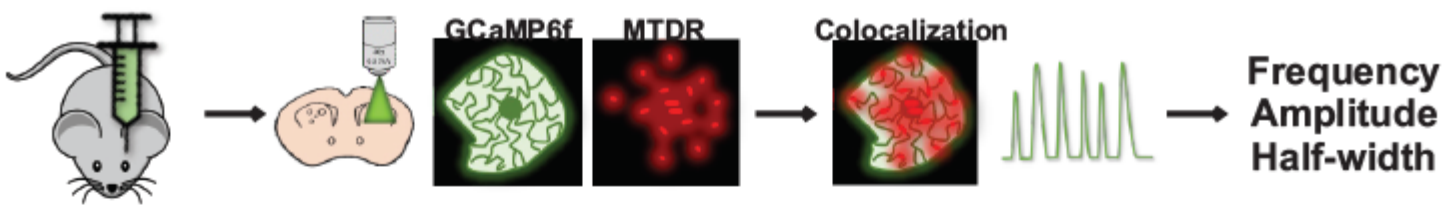

b Striatal Astrocyte
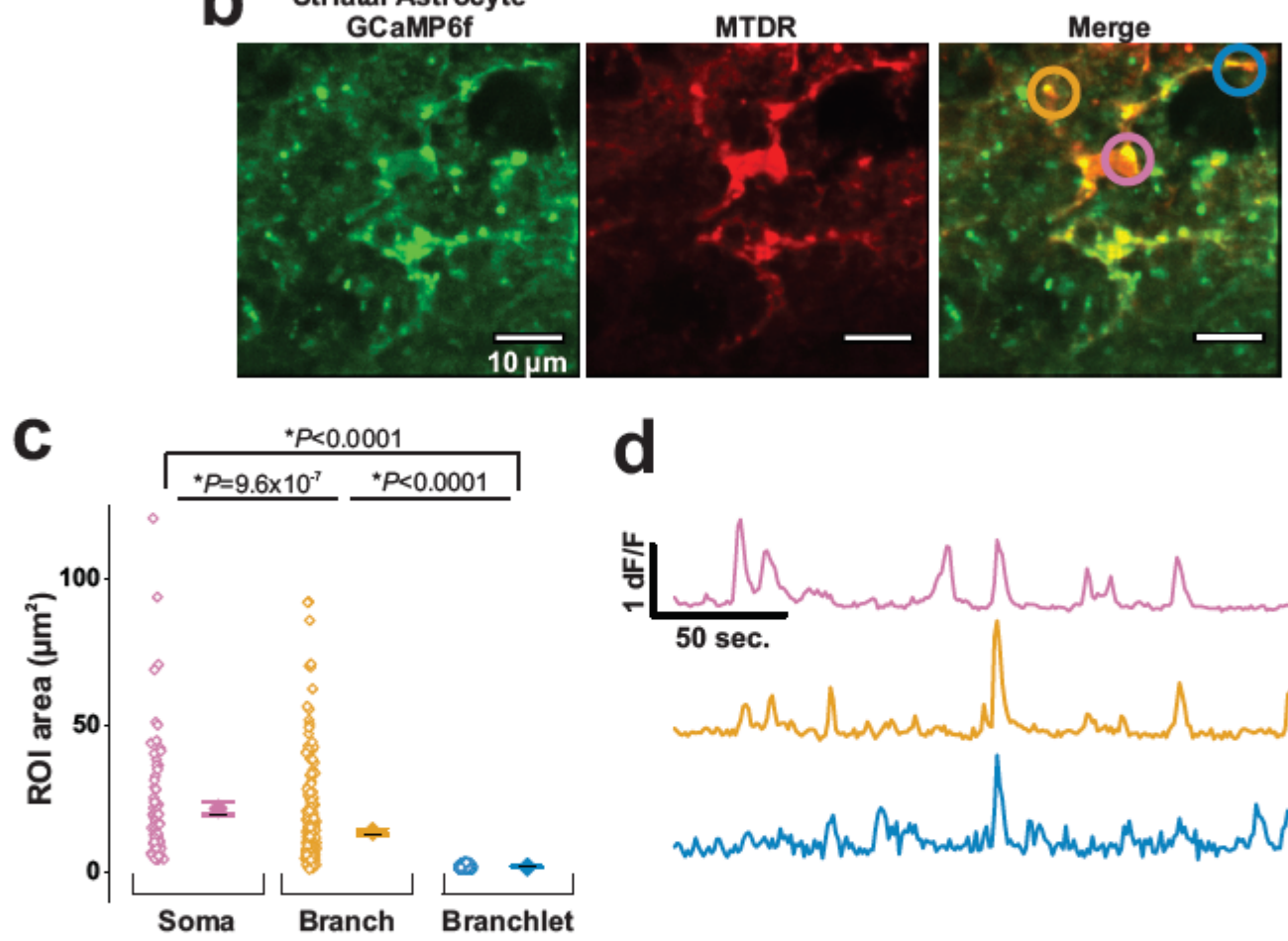

d
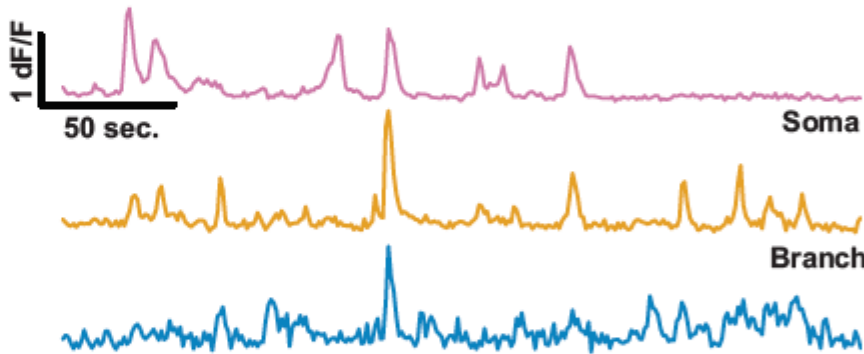

Branchlet
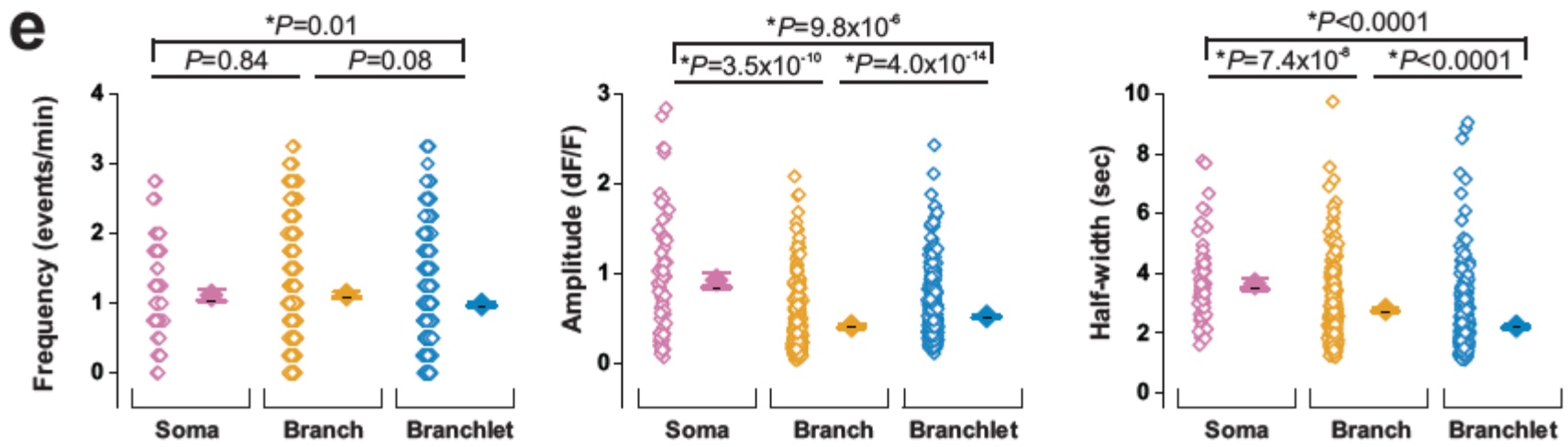

Figure 1

Spontaneous Ca2+ events in striatal astrocytic mitochondria visualized with AAV 2/5-GfaABC1D-mito-7GCaMP6f. a, Schematic of the GfaABC1D-mito-7-GCaMP6f construct and experimental approach. $b$, Representative time compressed confocal images of a striatal astrocyte expressing GCaMP6f (green) and MitoTracker Deep Red (MTDR, red) from live brain slices of WT C57BL/6 mice. Merged image shows somatic, branch, and branchlet mitochondrial regions of interest (ROIs) in magenta, orange, and blue 
circles respectively. Scale bar $=10 \mu \mathrm{m}$. c, Areas of somatic, branch and branchlet mitochondria in DLS astrocytes (56 astrocytes and 15 mice; $n=67$ somatic, 336 branch, and 605 branchlet mitochondria). $d$, Representative traces of spontaneous mitochondrial Ca2+ events in somatic (magenta), branch (orange) and branchlet (blue) mitochondria. e, Population data and mean values for spontaneous $\mathrm{Ca} 2+$ event frequency (left), amplitude (middle), and half-width (right) in striatal astrocytic mitochondria from 56 astrocytes and 15 mice ( $n=67$ somatic, 336 branch, and 605 branchlet mitochondria). Errors are \pm s.e.m. p-values are based on Mann-Whitney test.
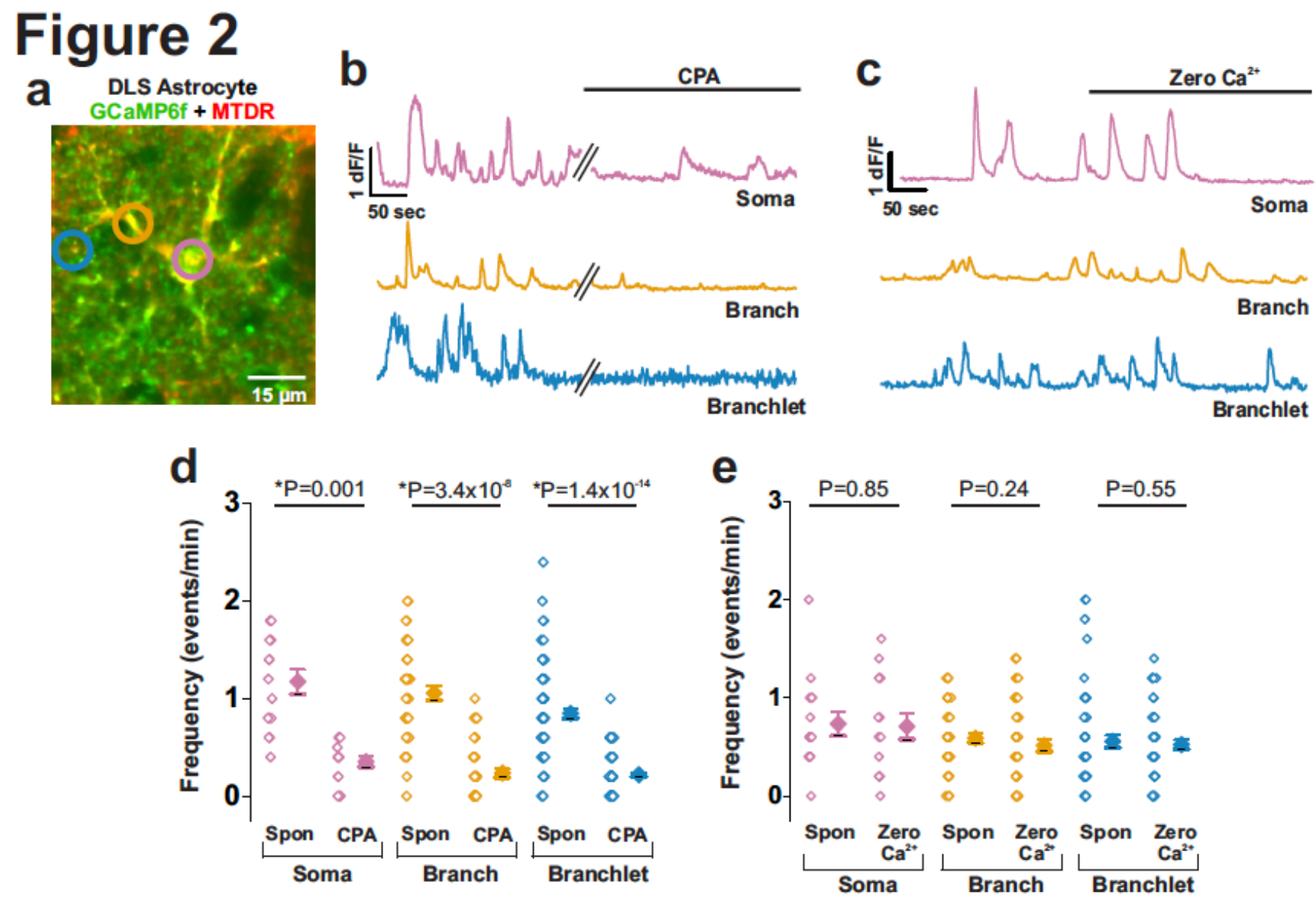

\section{Figure 2}

Spontaneous mitochondrial Ca2+ events in DLS astrocytes depend on ER- localized Ca2+ stores. a, Representative merged time compressed confocal image of a DLS astrocyte expressing GCaMP6f (green) and MTDR (red). Somatic, branch, and branchlet ROIs are shown by magenta, orange, and blue circles respectively. Scale bar $=15 \mu \mathrm{m}$. $\mathrm{b}$, Representative $\mathrm{Ca} 2+$ event traces of mitochondria in each subpopulation in response to CPA. c, Representative Ca2+ event traces from DLS astrocytic mitochondria before and after Zero Ca2+. d, Population data and mean values showing changes in Ca2+ event frequency in mitochondria before and after CPA (7 astrocytes and 4 mice; $n=15$ somatic, 43 branch, and 86 branchlet mitochondria). e, Ca2+ event frequency in mitochondria from each subpopulation before 
and after Zero Ca2+ (8 astrocytes and 4 mice; $n=12$ somatic, 45 branch, and 59 branchlet mitochondria). Errors are \pm s.e.m. p-values are based on Wilcoxon Signed Rank test for somatic mitochondria before and after CPA administration. All other data sets (CPA and Zero Ca2+) were subject to paired t-test.
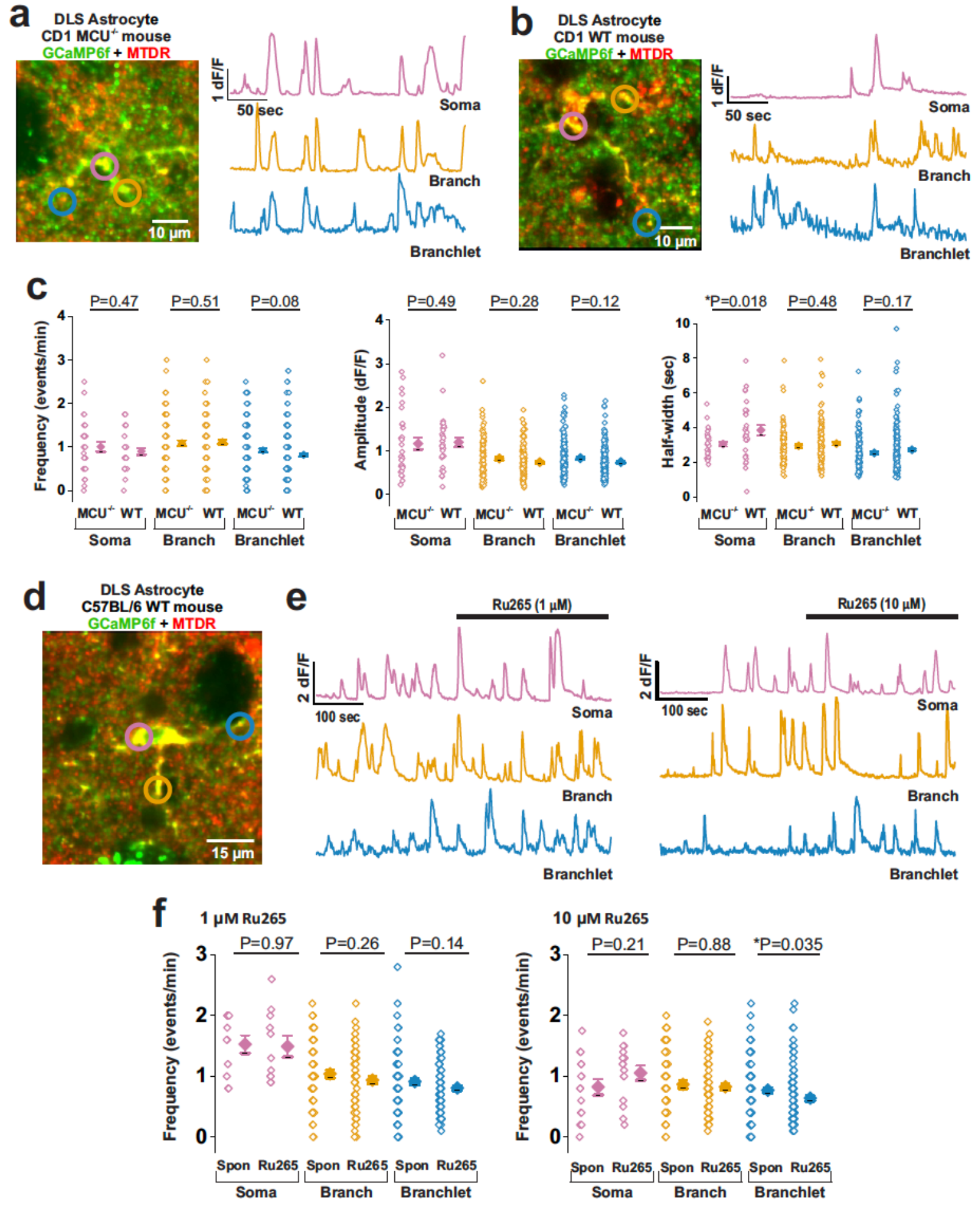

Figure 3

$\mathrm{Ca} 2+$ fluxes in astrocytic mitochondria do not depend on MCU. Representative merged time compressed confocal images of GCaMP6f + MTDR labeled DLS astrocytes and traces of spontaneous astrocytic 
mitochondrial Ca2+ events from a, CD1 MCU-/- and b, CD1 WT littermates. In each case, ROIs and corresponding traces for somatic (magenta), branch (orange), and branchlet (blue) mitochondria are shown. Scale bar $=10 \mu \mathrm{m}$. c, Population data and mean values comparing astrocyte mitochondrial Ca2+ event frequency (left), amplitude (middle) and half-width (right) (35 astrocytes and 5 MCU-/- mice; $n=39$ somatic, 99 branch, and 157 branchlet mitochondria, and 29 astrocytes from 4 WT littermates; $n=32$ somatic, 148 branch, and 236 branchlet mitochondria). Errors are \pm s.e.m. p-values are based on MannWhitney test. d, Representative time compressed confocal image of a DLS astrocyte during Ca2+ imaging with Ru265 application. Somatic, branch, and branchlet representative mitochondria are shown by magenta, orange, and blue circles, respectively. Scale bar $=15 \mu \mathrm{m}$. e, Representative Ca2+ event traces for somatic (magenta), branch (orange), and branchlet (blue) mitochondria with spontaneous Ca2+ events and after application of $1 \mu \mathrm{M}$ Ru265 (left) and $10 \mu \mathrm{M}$ Ru265 (right). f, Average Ca2+ event frequency of mitochondria before and after $1 \mu \mathrm{M}$ Ru265 (left graph) (7 astrocytes and 4 mice; $n=11$ somatic, 62 branch, and 80 branchlet mitochondria, and $10 \mu \mathrm{M}$ Ru265, right graph from 7 astrocytes and 4 mice; $n=$ 13 somatic, 57 branch, and 90 branchlet mitochondria). Errors are \pm s.e.m. p-values for comparison of MCU-/- and WT littermates are based on Mann-Whitney test. p-values for $1 \mu \mathrm{M}$ or $10 \mu \mathrm{M}$ Ru265 data are based on Wilcoxon Signed Rank test. 

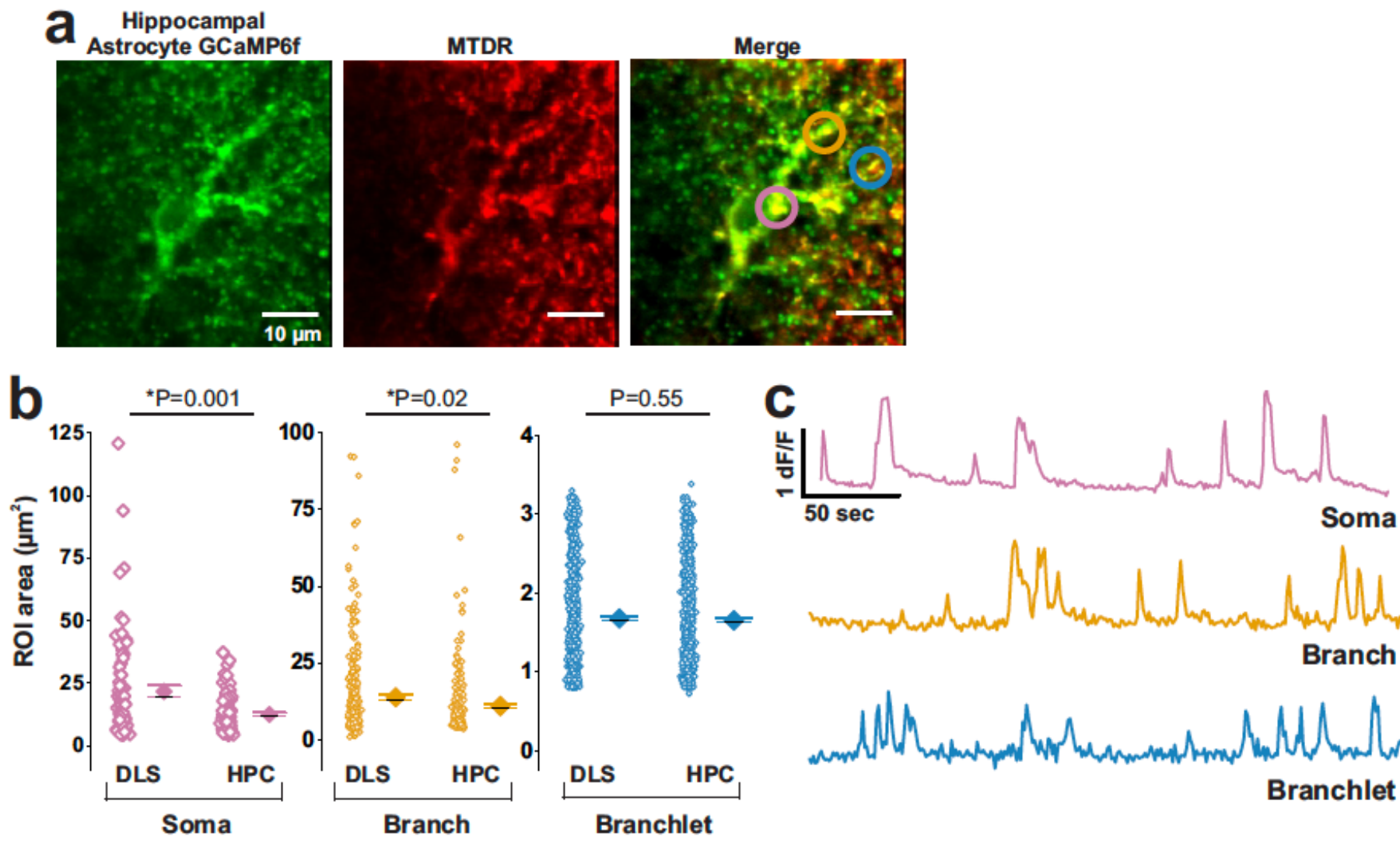

Soma
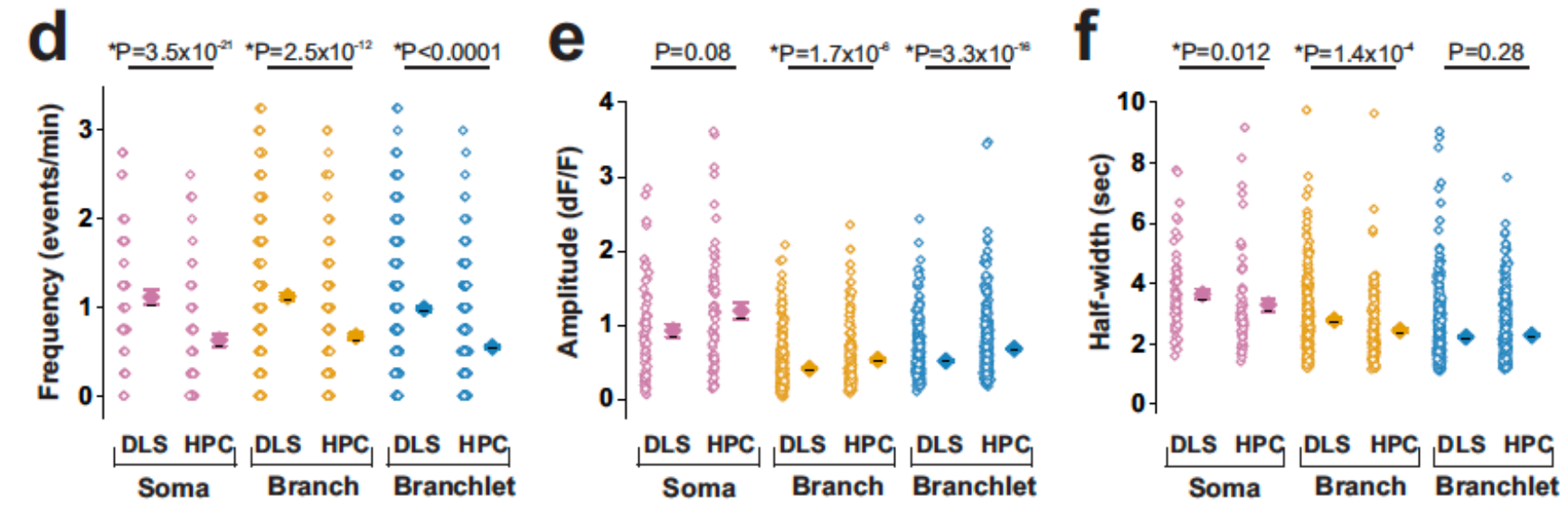

\section{Figure 4}

DLS and HPC astrocytes differ in mitochondrial sizes and Ca2+ event frequencies. a, Representative time compressed confocal images of a hippocampal (HPC) astrocyte from the CA1 region expressing GCaMP6f (green) + MTDR (red). Somatic, branch, and branchlet mitochondria are indicated by magenta, orange, and blue circles respectively. Scale bar $=10 \mu \mathrm{m}$. b. Population data and mean values comparing mitochondrial areas between 56 DLS astrocytes from 15 mice ( $n=67$ somatic, 336 branch, and 605 branchlet mitochondria) and 55 HPC astrocytes from 8 mice ( $n=79$ somatic, 211 branch, and 547 branchlet mitochondria). c, Representative traces of spontaneous mitochondrial Ca2+ events in somatic (magenta), branch (orange), and branchlet (blue) mitochondria of HPC astrocytes. d-f, Comparison of 
spontaneous astrocytic mitochondrial $\mathrm{Ca} 2+$ event frequency, amplitude and half-width between DLS and HPC astrocytes from 56 DLS astrocytes and 15 mice $(n=67$ somatic, 336 branch, and 605 branchlet mitochondria), and from 55 HPC astrocytes and 8 mice ( $n=79$ somatic, 211 branch, and 547 branchlet mitochondria). Errors are \pm s.e.m. p-values are based on Mann-Whitney test.

\section{Figure 5}

\section{a DLS Astrocyte - C57BL/6 WT mouse}

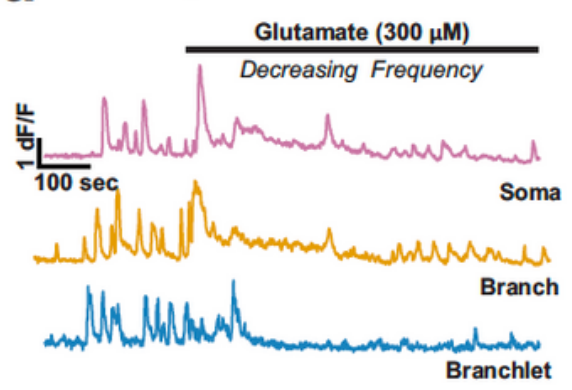

b

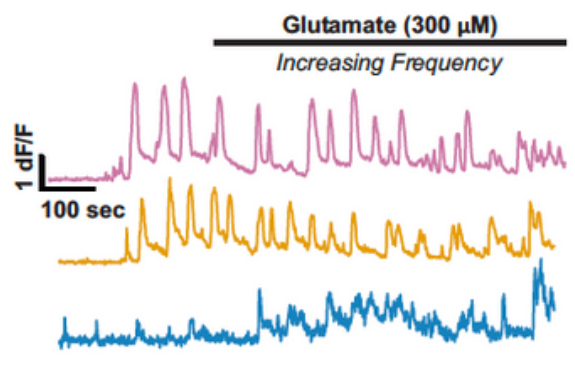

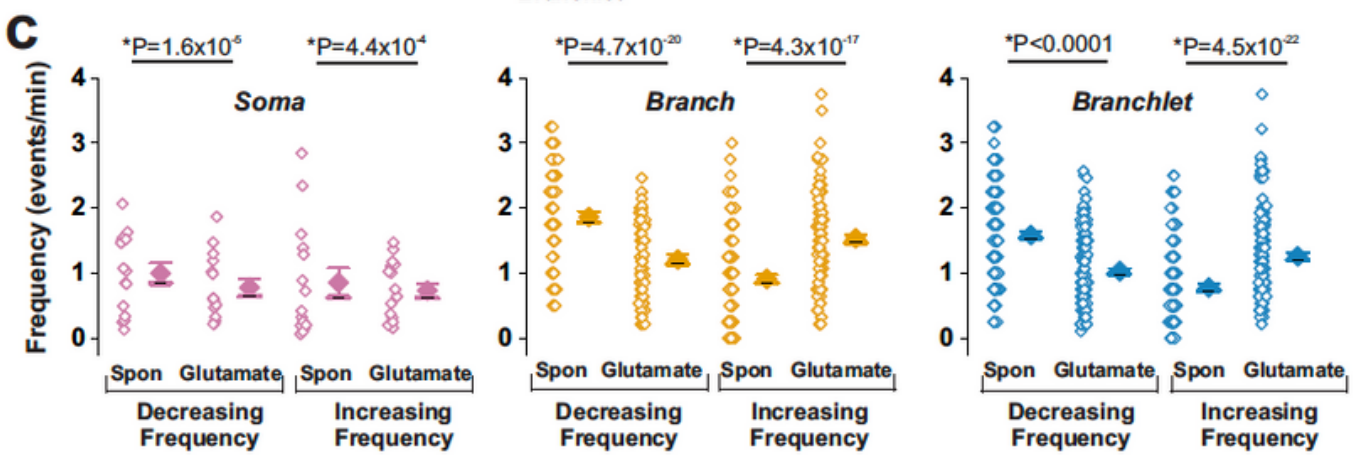

d HPC Astrocyte - C57BL/6 WT mouse

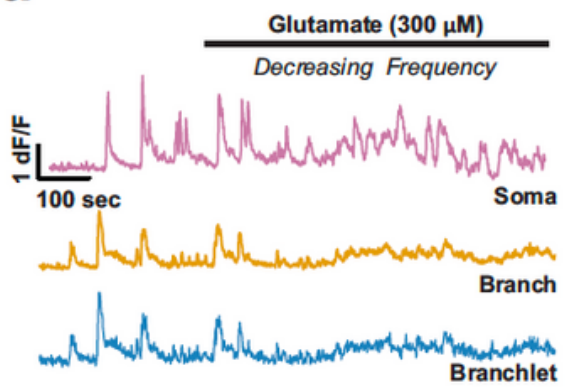

e

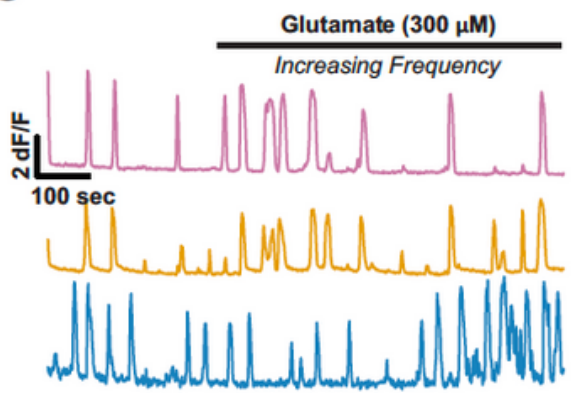

f

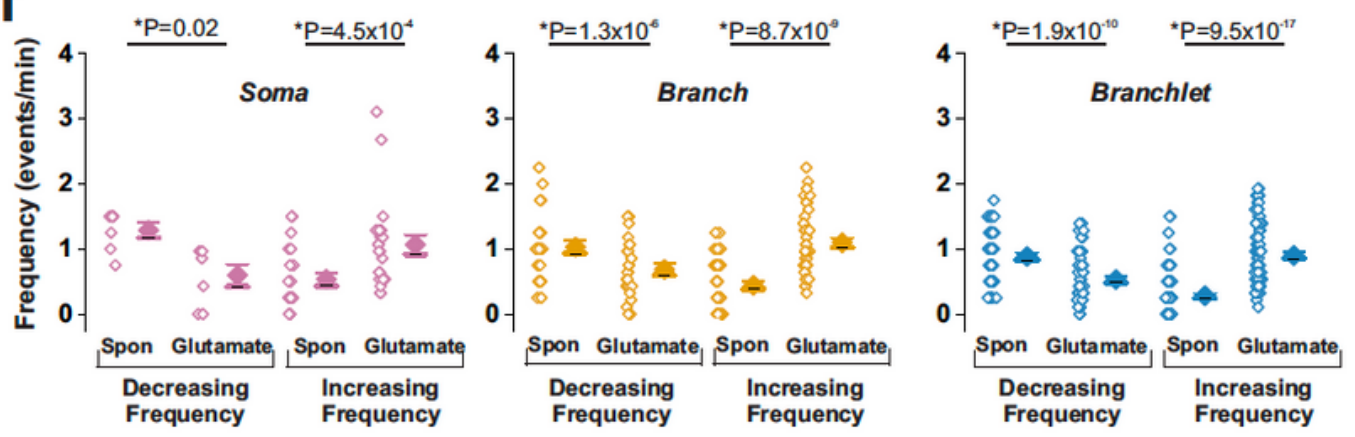

Figure 5 
Glutamate alters mitochondrial Ca2+ event frequencies in DLS and HPC astrocytes. Representative Ca2+ traces for somatic (magenta), branch (orange), and branchlet (blue) mitochondria from DLS astrocytes with either $a$, decreasing or $b$, increasing $\mathrm{Ca} 2+$ event frequency in response to glutamate. $c$, Population data and mean values for DLS mitochondrial Ca2+ event frequency from 23 DLS astrocytes and 12 mice. Mitochondria in graphs are segregated based on whether glutamate decreased or increased Ca2+ event frequency ( $n=15$ decreasing and 16 increasing somatic, 75 decreasing and 110 increasing branch, and 165 decreasing and 135 increasing branchlet mitochondria). d-e, As in a-b, but for HPC astrocytic mitochondria in response to glutamate. $\mathrm{f}$, As in $\mathrm{c}$, but for HPC mitochondria from 17 astrocytes and 8 mice ( $n=7$ decreasing and 21 increasing somatic, 25 decreasing and 44 increasing branch, and 59 decreasing and 97 increasing branchlet mitochondria). Errors are \pm s.e.m. p-values are based on paired sample t-test for decreasing and increasing frequency in DLS somatic mitochondria and decreasing frequency of DLS branch mitochondria. All other DLS data sets are based on Wilcoxon Signed Rank test. For data from HPC astrocytes, $p$-values are based on a paired t-test for decreasing frequency in HPC branch mitochondria, while all other data sets are based on Wilcoxon Signed Rank test. 
Figure 6
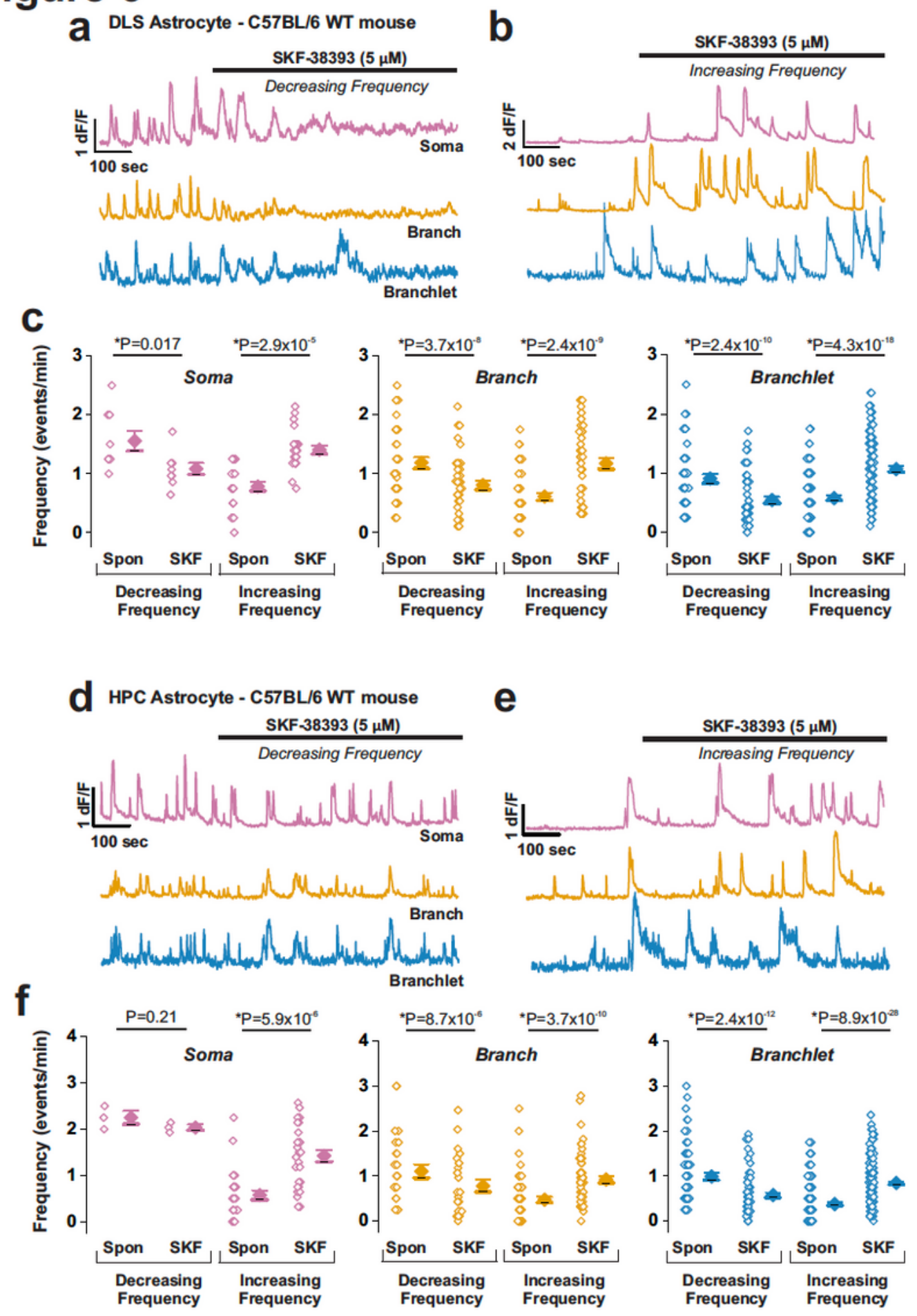

\section{Figure 6}

Responses of mitochondrial Ca2+ event frequency in DLS and HPC astrocytes to the D1-receptor agonist, SKF-38393. Representative Ca2+ traces for somatic (magenta), branch (orange), and branchlet (blue) mitochondria a, decreasing or b, increasing Ca2+ event frequency in response to SKF-38393. c, Population data and mean values for mitochondrial Ca2+ event frequency from 17 DLS astrocytes and 8 mice ( $n=9$ decreasing and 23 increasing somatic, 40 decreasing and 47 increasing branch, and 53 
decreasing and 105 increasing branchlet mitochondria) before and after SKF-38393. d-e, As in a-b, but for HPC astrocytic mitochondria with SKF-38393 application. f, As in c, but from mitochondria in 17 HPC astrocytes and 7 mice ( $n=3$ decreasing and 27 increasing somatic, 26 decreasing and 57 increasing branch, and 65 decreasing and 165 increasing branchlet mitochondria) before and after SKF-38393. Errors are \pm s.e.m. p-values are based on paired sample t-test for decreasing frequency in DLS somatic mitochondria. All other data sets for DLS and HPC astrocytes are based on Wilcoxon Signed Rank test.

\section{Figure 7}

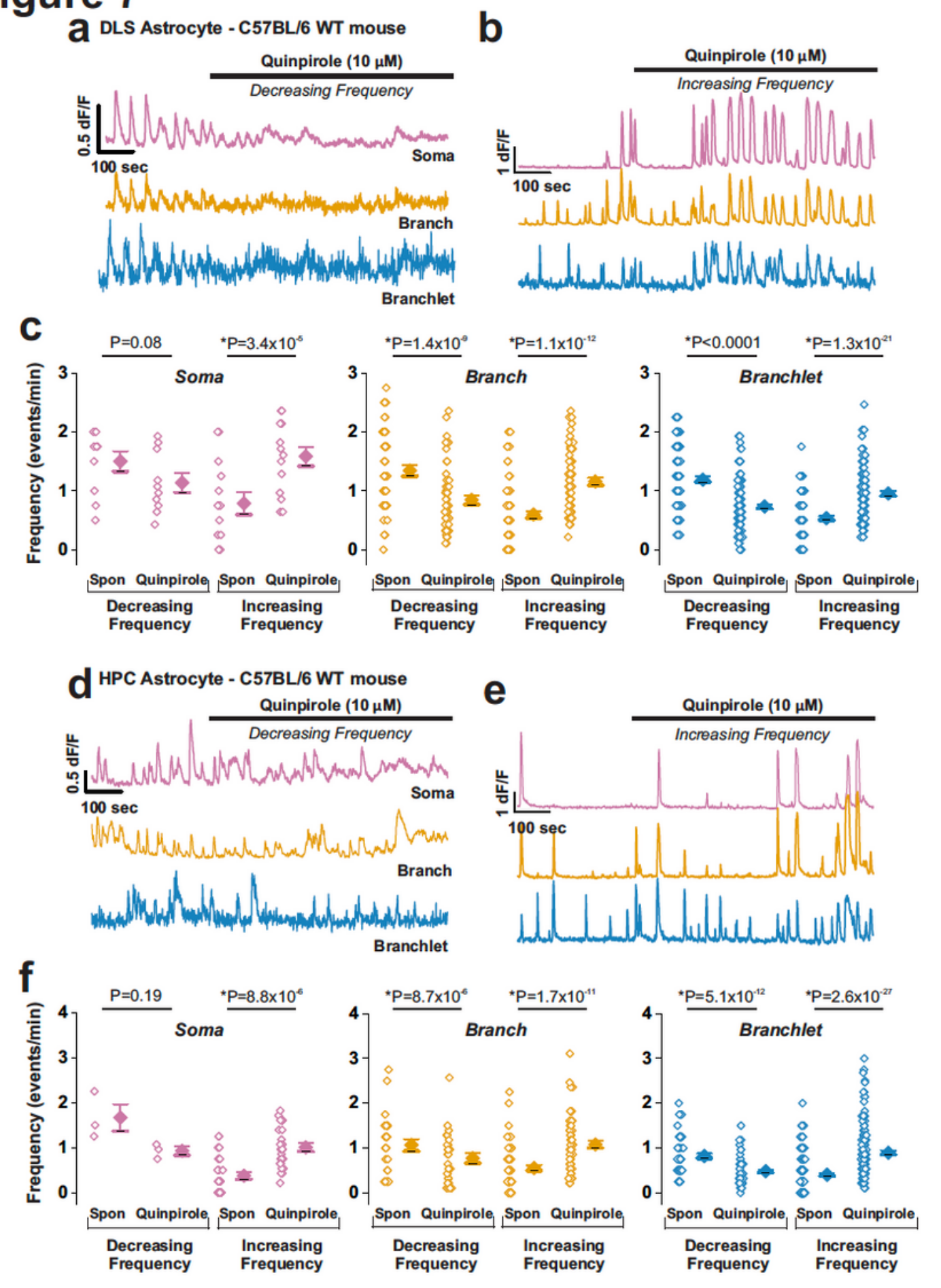


Responses of mitochondrial Ca2+ event frequency in DLS and HPC astrocytes to the D2-receptor agonist, quinpirole. Representative $\mathrm{Ca} 2+$ event traces for somatic (magenta), branch (orange), and branchlet (blue) mitochondria in DLS astrocytes in response to quinpirole $a$, decreasing or $b$, increasing $\mathrm{Ca} 2+$ event frequency. c, Population data and mean values for DLS mitochondrial Ca2+ event frequency changes in response to quinpirole from 21 DLS astrocytes and 11 mice $(n=10$ decreasing and 14 increasing somatic, 51 decreasing and 67 increasing branch, and 129 decreasing and 121 increasing branchlet mitochondria). d-e, As in a-b, but for mitochondria from HPC astrocytes. f, As in c, but for mitochondria from $16 \mathrm{HPC}$ astrocytes and 7 mice ( $n=3$ decreasing and 26 increasing somatic, 26 decreasing and 60 increasing branch, and 63 decreasing and 157 increasing branchlet mitochondria). Errors are \pm s.e.m. pvalues are based on paired sample t-test for decreasing and increasing frequency in DLS somatic mitochondria and decreasing frequency in HPC somatic mitochondria. All other data sets for DLS and HPC astrocytes are based on Wilcoxon Signed Rank test. 
Figure 8

a DLS - CD1 MCU' mice

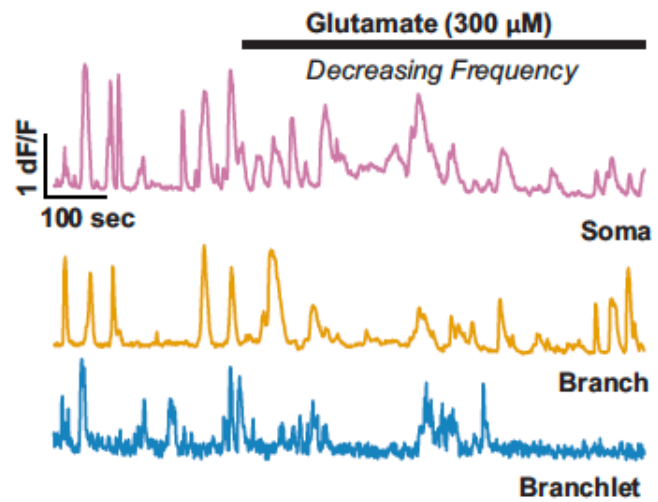

b DLS - CD1 MCU ${ }^{+}$mice

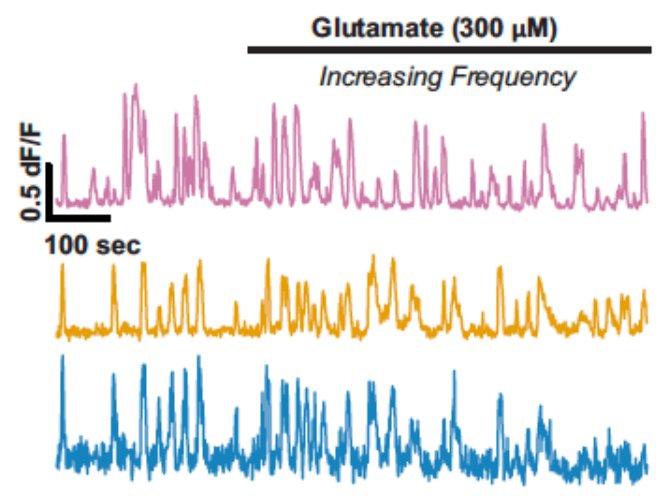

C DLS astrocytic mitochondria - $\mathrm{CD} 1 \mathrm{MCU}^{+}$mice

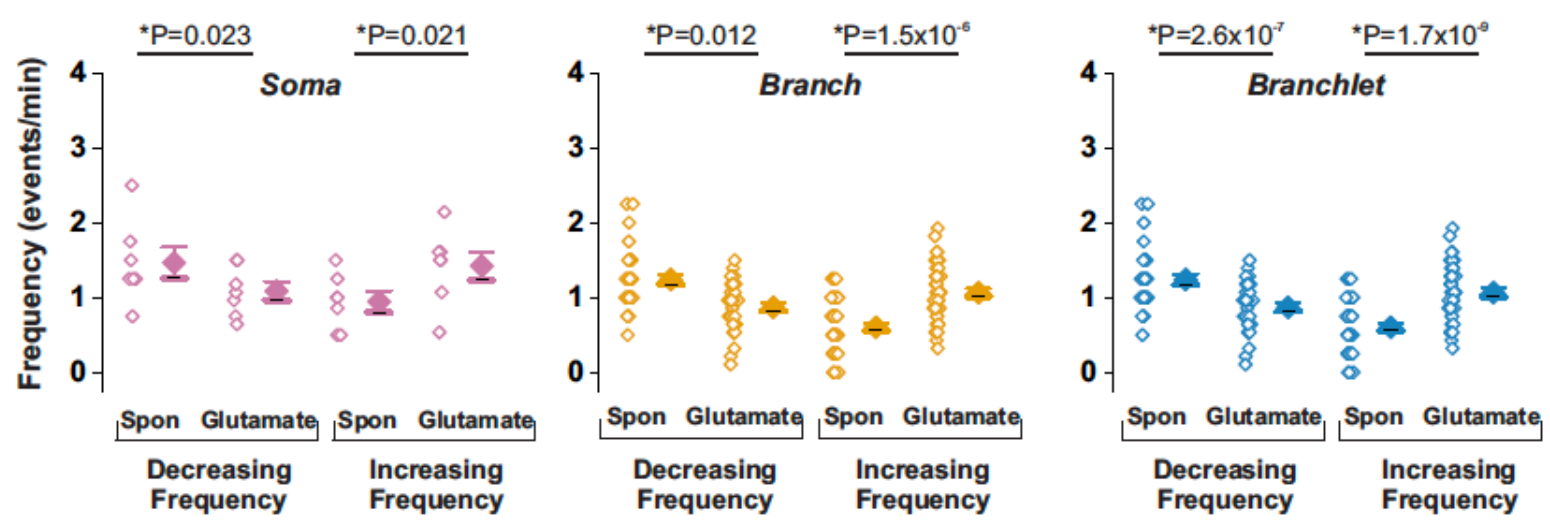

DLS astrocytic mitochondria - CD1 WT- mice

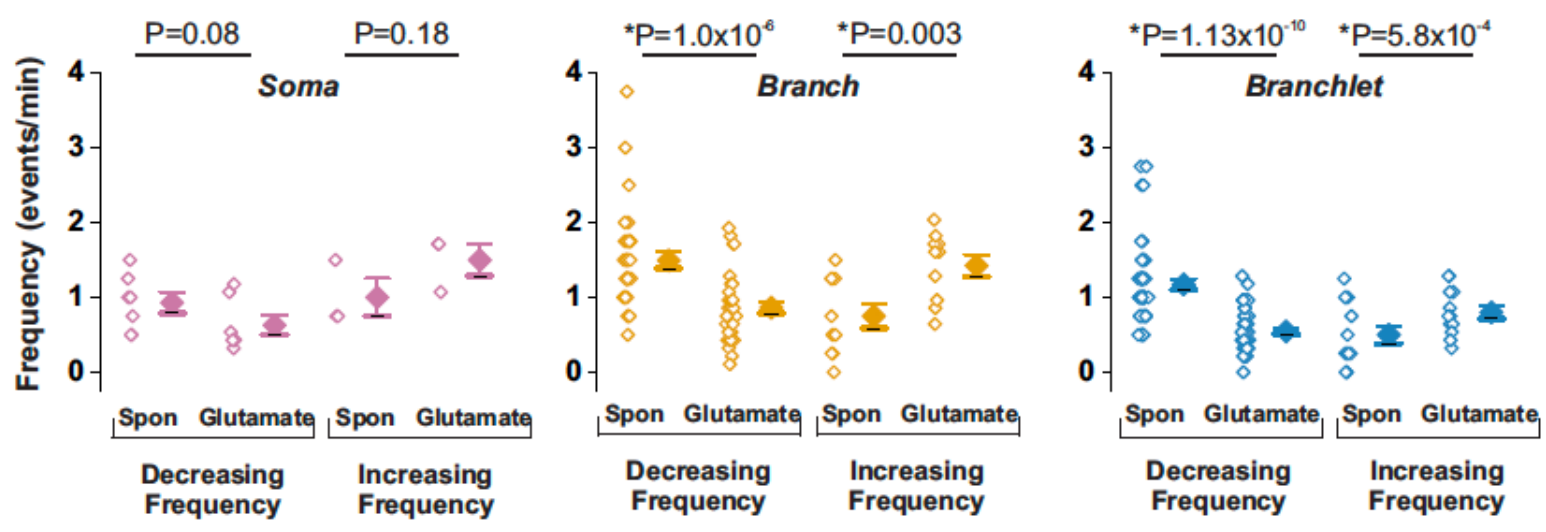

Figure 8

DLS astrocytic mitochondrial Ca2+ event responses to glutamate are not altered in MCU-/- mice. Representative Ca2+ event traces for soma (magenta), branch (orange), and branchlet (blue) mitochondria from DLS astrocytes of MCU-/- mice showing either $a$, decreasing or b, increasing Ca2+ event frequency in response to glutamate application. c, Population data and mean values for spontaneous and glutamate induced Ca2+ event frequency from 10 DLS astrocytes in 5 MCU-/- mice (n= 
7 decreasing and 10 increasing somatic, 18 decreasing and 18 increasing branch, and 35 decreasing and 48 increasing branchlet mitochondria). $d$, As in c, but for mitochondria from 10 DLS astrocytes in 4 WT littermates ( $n=3$ decreasing and 10 increasing somatic, 34 decreasing and 10 increasing branch, and 56 decreasing and 13 increasing branchlet mitochondria). Errors are \pm s.e.m. p-values for MCU-/- mice are based on paired sample t-test except that branchlet mitochondria were subject to Wilcoxon Signed Rank test. p-values for WT littermates are based on paired t-tests, except decreasing frequency branch mitochondria and decreasing frequency branchlet mitochondria which were subject to Wilcoxon Signed Rank test. 
a DLS - CD1 MCU' mice

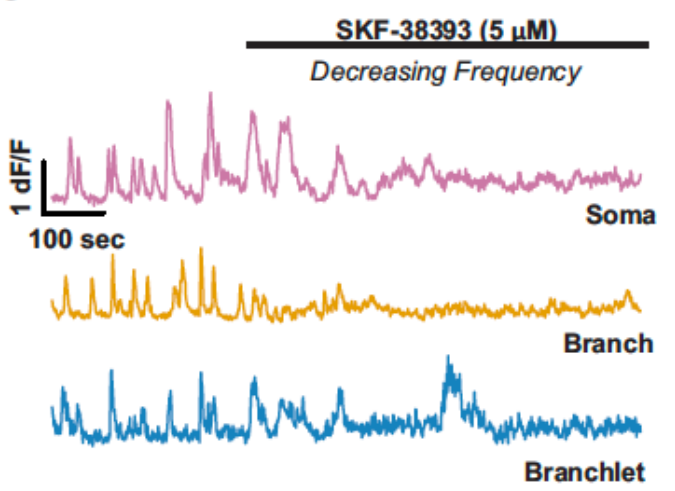

b DLS - CD1 MCU' mice

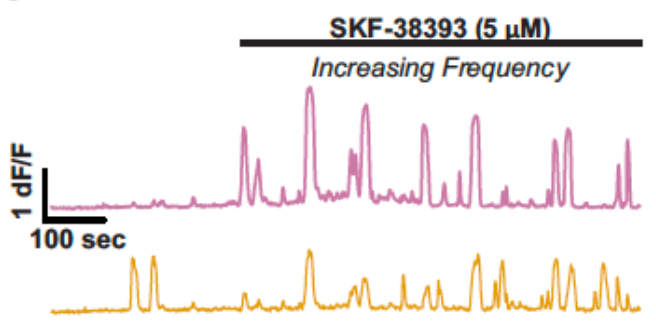

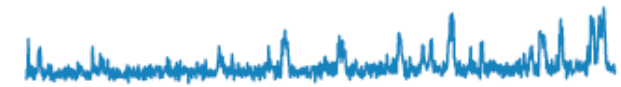

C DLS astrocytic mitochondria - CD1 MCU+ mice

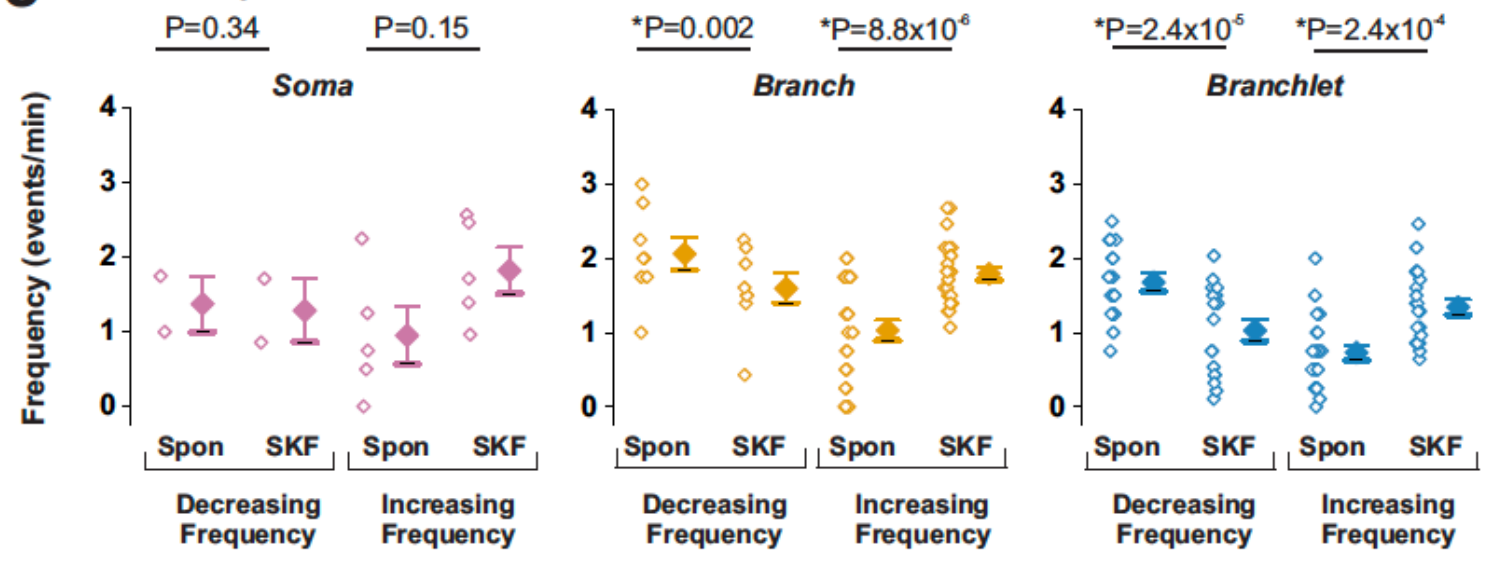

d DLS astrocytic mitochondria - CD1 WT- mice
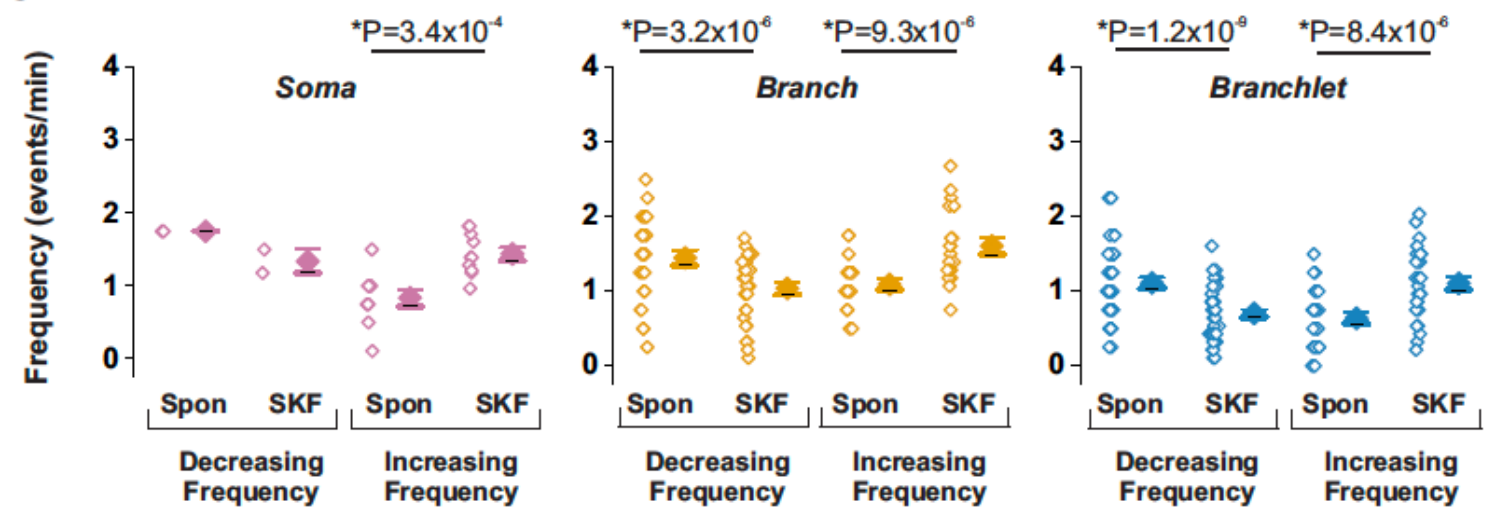

\section{Figure 9}

DLS astrocytic mitochondrial Ca2+ event responses to SKF-38393 are not altered in MCU-/- mice. Representative Ca2+ traces for somatic (magenta), branch (orange), and branchlet (blue) mitochondria from DLS astrocytes with either $a$, decreasing or b, increasing Ca2+ event frequency in response to SKF38393 application. c, Population data and mean values for spontaneous and SKF-38393 induced mitochondrial Ca2+ event frequency from 9 DLS astrocytes in 5 MCU-/- mice ( $n=2$ decreasing and 5 
increasing somatic, 8 decreasing and 26 increasing branch, and 17 decreasing and 21 increasing branchlet mitochondria). $d$, As in c, but for mitochondria from 9 DLS astrocytes in 4 WT littermates ( $n=2$ decreasing and 10 increasing somatic, 29 decreasing and 19 increasing branch, and 42 decreasing and 27 increasing branchlet mitochondria). Errors are \pm s.e.m. p-values for MCU-/- mice are based on paired ttests except for increasing frequency branch mitochondria which is based on Wilcoxon Signed Rank test. All p-values for WT littermates are based on paired t-test.

\section{Figure 10}

\section{a DLS - CD1 $\mathrm{MCU}^{+}$mice}

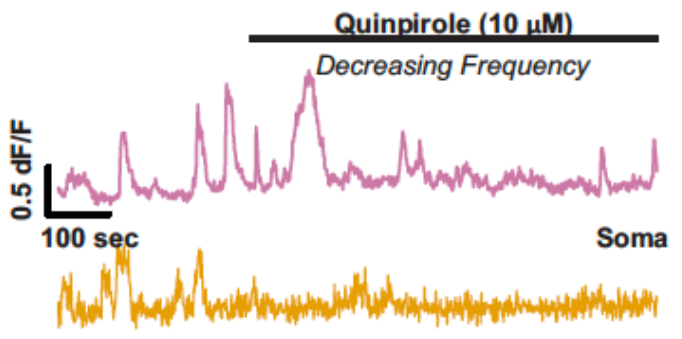

Branch

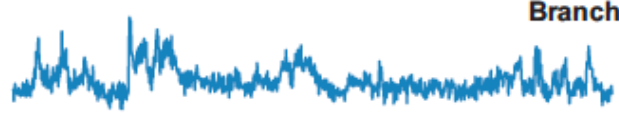

b DLS - CD1 $\mathrm{MCU}^{+}$mice

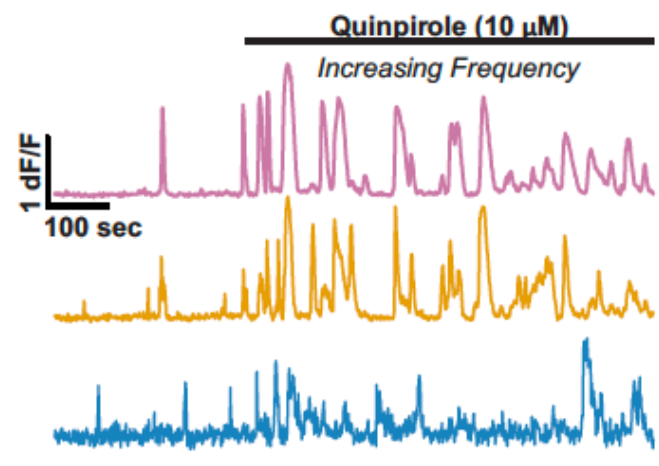

C DLS astrocytic mitochondria - $\mathrm{CD} 1 \mathrm{MCU}^{+}$mice

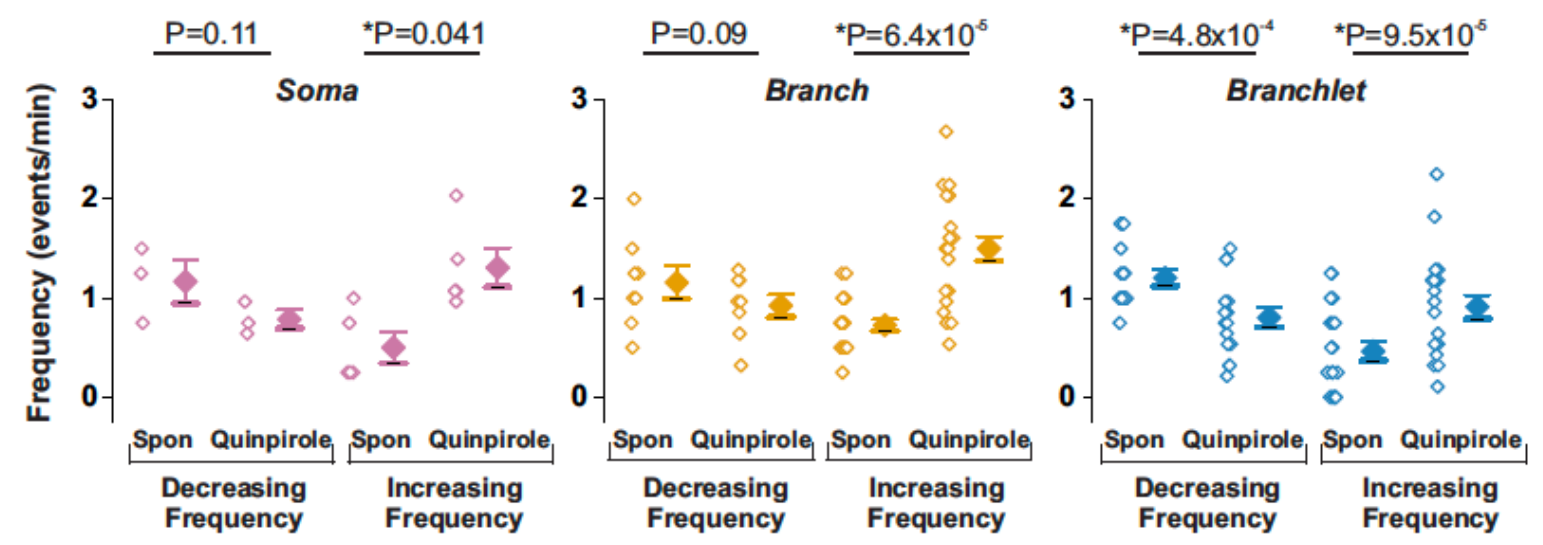

d DLS astrocytic mitochondria - CD1 WT- mice
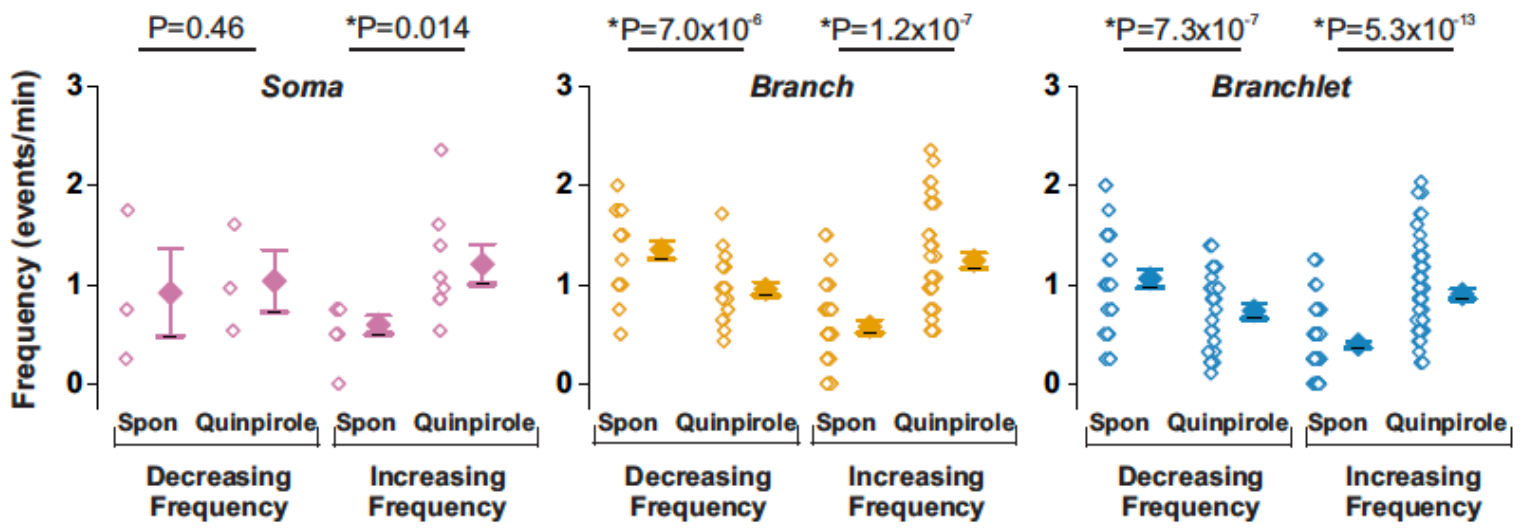
DLS astrocytic mitochondrial $\mathrm{Ca} 2+$ event responses to quinpirole are not altered in $\mathrm{MCU}-/$ - mice. Representative $\mathrm{Ca} 2+$ traces for somatic (magenta), branch (orange), and branchlet (blue) mitochondria from DLS astrocytes with either $a$, decreasing or $b$, increasing $\mathrm{Ca} 2+$ event frequency in response to quinpirole. $c$, Population data and mean values for changes in quinpirole induced Ca2+ event frequency from 9 DLS astrocytes in 5 MCU-/- mice ( $n=3$ decreasing and 5 increasing somatic, 8 decreasing and 21 increasing branch, and 16 decreasing and 20 increasing branchlet mitochondria). d, As in c, but for mitochondria from 10 DLS astrocytes in 4 WT littermates ( $n=3$ decreasing and 10 increasing somatic, 20 decreasing and 37 increasing branch, and 29 decreasing and 69 increasing branchlet mitochondria). Errors are \pm s.e.m. p-values for MCU-/- mice are based on paired sample t-test, except for branch mitochondria with increased in frequency and branchlet mitochondria with decreased or increased in frequency, that were subject to Wilcoxon Signed Rank test. p-values for WT littermates are based on paired sample t-test, except for increasing frequency branch mitochondria and increasing frequency branchlet mitochondria which were subject to Wilcoxon Signed Rank test.

\section{Supplementary Files}

This is a list of supplementary files associated with this preprint. Click to download.

- SupplementarylnformationTEHRSfinal.pdf

- SupplementarymoviecaptionsTEHRSfinal.pdf

- SupplementaryMovie1.avi

- SupplementaryMovie2.avi

- SupplementaryMovie3.avi

- SupplementaryMovie4.avi

- SupplementaryMovie5.avi 